\title{
Development of an Explicit Multigrid Algorithm for Quasi-Three-Dimensional Viscous Flows in Turbomachinery
}

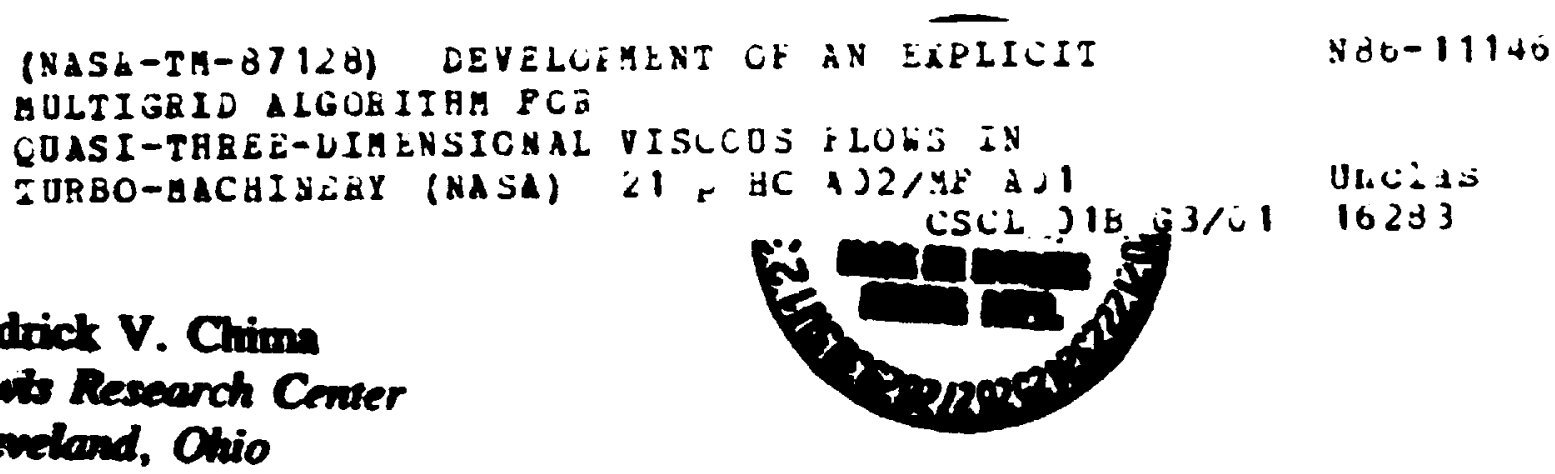

Prepared for the

Twenty-fourth Aerospecas Sciences Meeting sponsored by American Institute of Aeronautics and Astronautics Reno, Nevada, Januery 6-9, 1986 
Roorick V. Chima

National Aeronautics and Space Administrat Lewis Research Center Clevelano, Onio 44135

\section{Abstract}

A rapid quasi-three-dimensional analysis has been ceveloped for blade-ta-blade llows in turbomachinery. The analysis solves the unsteady Euler or thin-layer Navier-Stokes equations in a body$f i+i e d$ coordinate system. It accounts for the effects of rotation, raoius change, and streamsurface thickness. The Balowin-Lomax eddyviscosity model is used for turbulent flows. The equations are solved using a two-stage Runge-kutta icheme made efficient oy use of vectorization.d variable time-step, and a fiux-tased multigrid scheme, which are all described. A stability andysis is presented for the two-stage scheme. Results ior flat-Dlate model problem stow the applicao:lit, of the metnoo to axial, racial, ano rutating geometries. Resuits for a centrifugal impeller and radial oiffuser show that the quasi-three-simen inal viscous analysis can bes practical oesign ?.

\section{Introduction}

Turbomacninery inienced to produce large amounts of power from small volume of ten require use of radial-flow or mixeu-flow components, that is, components in which the streanu ise velocity is not strictiy axial. Radial-flow turbomachines such as centrifugal imuellers, radial diffusers, and radial-inflow turbines nave a prerominantly radial flow oirection. Mixed-flow turbomachines may de used wnen restrictions on space prevent a completely radial flow. Complicated geometries. snock waves, and viscous onenomend make analys is of radial-or mixeo-flow turbomachines more aifficuit than andysis of strictly axial-flow macnines.

Anslysis of axial-flow turbomachinery blade rows is usually simplified by modeli ing a blade section as a flat cascade. The governing cquations for a flat casidode are the same twooimensional fow equations that are solveo for isolateo airfoils. so cascade analyses often iraw neavily on numerical techniques developed for iwo-aimensional external fiows. Examples of fla: cascade analyses incluce panel methads. Dotential methods, Euler solutions, 3.4 ano Navier-

flows in radial-or mixed-flow turbomachines are inherently three-gimensional, requiring specification of the axial, radial, and tangential velocity components to fuliy specify the flow. A simplification that allows these machines to be andlyzeo in two ginensions was proposec oy C.H. Wu in $1952 .{ }^{\circ}$ In wu's model the flow is assumed to follow on axisymetrtc stream surface (Wu's "S2 surface." Fig. I). The radius and thickness of the stream surface are assumeo to be krow as functions of the streamu ise distance. These quantities are usually obtained from an axisymetric through-flow or "mericional" and lysis, ${ }^{B}$ somgtimes coupled with a boundary
layer and lys is 9 on the hud and shroud.

The equations governing the flow along the stream surface combine the axial-and radialvelocity components into one streamw ise component. and are thus two-dimensional. The solution can be resolveo into three velocity components since the shape of the surface is known. Specifying the strean-surface thickness allows variable blace heights and end wall displacemer: thicknesses to be mosclled. This is similar to specifying area change in the one-dimensional nozzle equations. since the effects of racius change and streanisurface thickness are modellea in illis analys is, if is termed "quasi-three-oimensional." Examples of quasi-three-aimensipgal turbomachinery andyses incluoe gane? methoos, 10 stream-function methocs. Potential hetnocs, It ano Euler methocs. 13

In the present work, the Euler ano kavierStokes code develooeo for flat cascaces in Ref. 6 $n$ as been extendeo to a quasi-tnree-oimensianal analysis. It is thrught that this is the first Navier-Stokes andysis to include the effects of rotation, radius change, and stream-surfage thicknegs. The explicit MacCormack algorithm used in ${ }^{6}$ has been replaceo with an explicit two-stage Runge-kutta finite-djfference algorithm odsed on the work of Jamusc: 5 Efficiency is achieved by :hree means: vectorizat: 1 . use of a variable time-step, ?ho by use of a multigrio scheme oeveloped by $i 1$ ? and mucified by johnson and in ima. $17-19$

\section{Goverri:nc Equations}

The axisymetric $(m, \theta)$ coorainate system us.20 for the suasi-three-oimensional analys is is show in $f i g$. I. Here the m-coordinate is defin 0 oy

$$
a^{2}=0^{2}+a^{2}
$$

and the -coordinate is defined oy:

$$
0 . \dot{s}^{\prime}-\ldots t
$$

where $\theta^{\prime}$ is fixed in snace and $e$ rotates with the blace row with angular velecity 8 . The radius $r$ and the stream surface thickness $h$ are taken to be known functions of $m$. In this cooroinate system the oimerisionless Navier-Stokes equations may be written in the following nearlyconservative form:

$$
a_{t} Q+a_{m}\left(F-R e^{-1} R\right)+\partial_{\theta}\left(G-R e^{-I_{S}}\right)=K
$$


where

$a=r n\left[\begin{array}{l}0 \\ o v_{m} \\ o v_{\theta} r \\ e\end{array}\right]$

$F=r n\left[\begin{array}{l}o v_{m} \\ o v_{m}^{2}+p \\ \left(o v_{m} v_{0}\right) r \\ v_{m}(e+p)\end{array}\right]$

$G=n\left[\begin{array}{l}0 w_{\theta} \\ 0 v_{m} w_{\theta} \\ \left(0 v_{0} w_{0}+p\right) r \\ w_{3}(e+0)+m_{i p}\end{array}\right]$

$k=r n\left[\begin{array}{l}0 \\ k_{2} \\ 0 \\ 0\end{array}\right]$

$R=r n\left[\begin{array}{l}0 \\ a_{11} \\ 0_{12} r \\ R_{4}\end{array}\right]$

$S=n\left[\begin{array}{l}0 \\ 0_{12} \\ 0_{22} r \\ s_{4}\end{array}\right]$

ana

$w_{0}=v_{\theta}-n$ is the relative tangential velocity component.

$e=o\left[C_{Y}^{\top}+1 / 2\left(v_{i}^{2}+v f\right)\right]$ is the total energy per unit volume,

$\left.p=(r-1)\left[e-1 / 2(v\}+v_{f}^{2}\right)\right]$ is the pressure,

$k_{2}=\left(o v_{0}^{2}+p-o_{22}\right) r m / r+\left(c-o_{33}\right) \mathrm{hm} / \mathrm{h}$

$r_{m} / r=\frac{1}{r} \frac{d r}{\partial m}$

$n_{m} / n=\frac{1}{n} \frac{c n}{d r}$

The viscous terrs in ine enercy equation are:

$k_{4}=\frac{h}{(r-i) P_{r}}\left(a_{n} d^{i}+v_{\pi i}^{0} 11+v_{0}{ }^{0} 1 i\right)$

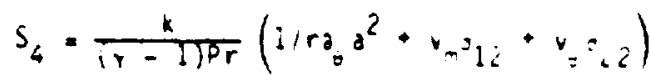

$15 ;$

where

$a=\sqrt{T D / C}$ is the soric velucity, anc the normalized thermal conouctivity $k$ equals one.

The shear stress terms are:

$$
\begin{aligned}
& 0_{11}=2 a_{m}{ }_{m}+17 \cdot \bar{v} \\
& a_{22}=2 u\left(a_{0} v_{\theta}+v_{m} r_{m}\right) / r+28 \cdot \bar{v}
\end{aligned}
$$

$$
\begin{aligned}
& \sigma_{33}=2 \omega v_{m} n_{m} / n+\lambda \theta \cdot \nabla \\
& \sigma_{12}=u\left(\partial_{r_{1}} v_{\theta}-v_{\theta} r_{m} / r+1 / r a_{\theta} v_{m}\right)
\end{aligned}
$$

and

$$
\lambda \theta \cdot \dot{v}=-\frac{2}{3}+\left[\partial_{m} v_{m}+v_{m}\left(r_{m} / r+n_{m} / n\right)+1 / r \partial_{\theta} v_{\theta}\right]
$$

is the dilitation.

The equations are nonaimensionalized by arbitrary reference quantities inere the inlet total density and critical sonic velocity define the reference state), and the Reynolas number $R e$ ano the tranotl number $\mathrm{Pr}$ must be specified in terms of tuat state. These equations assume that the specific neats $C_{p}$ and $C_{v}$ and the Pranot 1 number are constant, that stokes riypothes is $\lambda=-2: 3 \mathrm{w}$ is valio, ano that the effective viscosity may be written

$$
\omega=\omega_{\text {lamirar }}+\text { turoulent }
$$

Equatiuns (3) to (6) are transformed from the $(m, 0)$ cocroinate system to a ceneral bocy-fitted $(\xi, n)$ crordinate systeri using standard methods. The trin-layer assumption is then used to eliminate viscous derivitives in the streamwise ( $\xi$ ) oirection, thereby redusing computational overhead while retaining the capability of computing separated flows. The resulting equations are similar to those ceveloped by katsanis.20

$$
\partial_{t} \hat{q}+\partial_{\hat{q}} \hat{F}+\partial_{n}\left(\hat{G}-2 e^{-1 \hat{S}}\right)=\hat{k}
$$

where

$\hat{a}=j-1\left[\begin{array}{c}c \\ \therefore v_{m} \\ i v_{v} r \\ i\end{array}\right]$

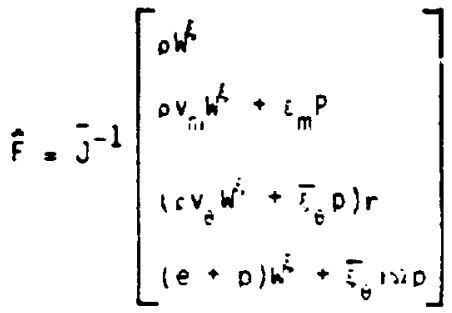

$i=i-i\left[\begin{array}{l}a \\ a_{2} \\ \vdots \\ i\end{array}\right]$

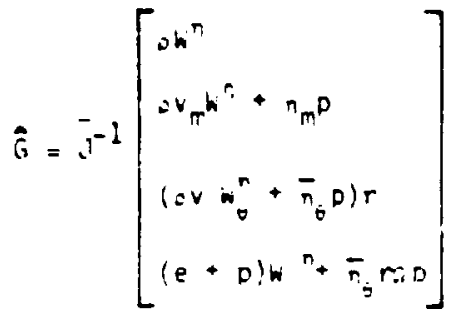

$\dot{S}=j^{-1}\left[\begin{array}{l}r \\ r^{0} 1 !+\ddot{n}_{b} c_{12} \\ 1 r_{\pi^{0} 12}+\bar{n}_{i} o_{22} i r \\ s_{4}\end{array}\right]$ 
and

$K_{2}=\left(O v^{2}+p-R e^{-1} o_{22}\right) r_{m} J r+\left(p-R e^{-1} o_{33}\right) h_{n} / h$

$s_{4}=\frac{k}{r-I T P_{r}}\left(n_{m}^{2}+\frac{\eta^{2}}{\eta_{0}}\right) n_{n} a^{2}+v_{m} s_{2}+v_{0} s_{3}$

In Eq. (8) the overbar: denote a rescaling of the metric terms:

$$
\bar{c}_{0}=c_{0} / r ; \quad \bar{n}_{0}=\eta_{1} / \mathrm{r}: \quad \bar{j}^{-1} \cdot r h j^{-1}
$$

where $)$ is the Jacosian of the transformation

$$
J=6 m_{0}^{n}-n_{m} b_{0}=\frac{1}{\left(m_{n} n_{n}-m_{n} n_{\varepsilon}\right.}
$$

and the metric quantities are determined from the grid-point coordinates using central aifferences and:

$$
\begin{array}{ll}
\varepsilon_{m}=J_{n} ; & \varepsilon_{0}=-J n_{n} ; \\
n_{m}=-J_{6} ; & n_{0}=J m_{c}
\end{array}
$$

The relative contravariant velocity compunents $W^{*}$ and $W^{n}$ long the 6 and $n$ grid lines are given by:

$$
w=\varepsilon_{m} v_{m}+\bar{\tau}_{0} w_{0} ; \quad w^{n}=n_{m} v_{n}+\bar{n}_{0} w_{0}
$$

The shear stress terms are found from Eq. (6) by replacing $a_{m}$ with $n_{m} n_{n}$ and $1 / r g_{\text {g with }}$ $n_{n} \cdot n$.

The quasi-three-dimensional equations (Eqs. (2) to (12)) are similar to the twooimensional equations solved in Ref. 6 except for the source term $K_{2}$, the radius appearing in the - -momentum equation, the rescaled metrics

([q. (9)), and the relative velocity component W appearing in the contravariant velocities (Eq. (12)). Equations (7) to (12) reduce to the two-dimensional equations for constant $r$ and $n$, and zero rotation. Note that EqS. (7) to (12) are independent of the magnitude of the stream surface thickness $h$ so that any function $h(m), 0$ may be used. The equations oo depend on the magnitude of the radius $r$ because of the $1 / r$ terms scal. ing 5, and in Eq. (9).

For turbulent flows the two-layer eddyviscosity model developed by Baldwin and Loma $x^{21}$ is used. In the $(m, 0)$ coordinate system the wall shear $I_{W}$ and vorticity a required by the model are given by

$$
\begin{aligned}
T_{w}=0_{12 w} & =\left(\partial_{m} v_{0}+1 / r_{0} v_{m}-v_{0} r_{m} / r\right) w \\
w & =\frac{1}{2}\left(\partial_{m} v_{0}-1 / r_{0} v_{m}+v_{0} r_{m} / r\right)
\end{aligned}
$$

\section{Computational Grid}

Body-fitted grids for this work were generated using the GRAPE code (GRios about Airf Jlls using $\mathrm{Zgisson's} \mathrm{Equaticn)}$ developed by Sorenson.22, 23 Briefly, the code allows arbitrary specification of inner and outer boundary points, then generates interior points as solution to Poisson equation. Forcing terms in the Poisson equation are chosen such that desired gris spacing and intersection angles may be maintained at the inner ans outer boundaries.

New inner and outer boundary subrout ines were written for turbomachine geanetries. The new inner bounoary has constant spacing around blade leacing and tialling edges, larger constant spacing over blade surfaces, and exponential stretching connecting the regions. The new outer boundary is composed of the meari-camber line between the blades. quaratic extension upstream, and linear extension downstream. The C-snaded grids are periodic over the pitch of the blade. Grids are generated in a Cartesiar. $\left(m, F_{4}\right.$; coorinate system, where $F$ is some mean adlus. The local radius and stream surface tilickness are Suppiied to the Navier-Stokes =uue at a later time as tabulated functions of $A$, then spline-fit on to the grid. The ....s $r_{m} / \vec{r}$ and $h_{m} / h$ in Eas. (7) and (8) are calculated using central differences and are stored.

\section{Initiul Conditions}

Since a centrifugal compressor can produce pressure ratios of $5: 1$ or greater it is not generally possible to start a quasi-three-dimensional calculation with conctant initial conditions. Instead an andytic solution of the one-dimensional flow equations with area change is used. Turbomachinery blades are usually designed based on desired ledoing- and trailing-edge veloclty triangles, so the relative flow velocity $W$ and angle a at the leading edge and the relative flow angle at the trailing edge art used as input for the initial conditions.

Using ()' to denote absolute total condi: lons and ()" to denote relative total conditions, the continulty ano energy equations are

$$
\begin{gathered}
\dot{m}=\text { orhsow cos } a=\text { constant } \\
1=C_{p} T^{\prime}-r_{0} v_{\theta}=C_{p} T^{\prime \prime}-1 / 2 r^{2} \theta^{2}=\text { constant }
\end{gathered}
$$

where $\Delta \theta$ is the blade spacing and $I$ is the rothalpy.

Dutside of the hlade row the angular momentum equation gives:

$$
r v_{\theta}=\text { constant }
$$

Equations (16) and (17) can be used with the isentropic relations 20 show that total conditions are corstant outside of the blade row. Evaluating EQ. (15) at some point $1 / 2$ and using $E Q$. (17) to eliminate a gives: 


$$
w_{?}^{2}=\left(\frac{\dot{m}}{\rho_{2} r_{2}^{h}{ }^{\Delta \theta}}\right)+w_{\theta 2}^{2}
$$

Now the isentropic relations and the defini.. tion of $\mathrm{T}_{2}$ " can be used to eliminate ${ }_{2}$ giving:

$$
p_{2}=o_{2}^{\prime \prime}\left(1-\frac{w_{2}^{2}}{2 C_{p} T_{2}^{*}}\right)^{\frac{1}{r-1}}
$$

where

$$
\rho_{2}^{\prime \prime}=\rho_{1}^{\prime \prime}\left(\frac{T_{2}^{\prime \prime}}{T_{1}^{n}}\right)^{\frac{1}{r-T}}
$$

Substituting Eq. (19) into Eq. (18) gives:

$$
w_{2}^{2}-\varphi_{2}^{2}\left(1-\frac{w_{2}^{2}}{2 C_{p} T_{2}^{w}}\right)^{\frac{-2}{r-1}}-w_{02}^{2}=0
$$

where

$$
\nu_{2}=\frac{\dot{m}_{1}^{\prime \prime}}{D_{2} r_{2} h_{2} \Delta \theta}
$$

Equation (20) is solved for $W_{2}$ at each grio point upstream of the blade using Newton iteration. Other flow quant ities ara then found using Eq. (17), the knowr total conditions, and

Withing the blade row $r v_{8}$ \& constant, so Eq. (17) is replaced with an assumption that the flow angle of varies linearly through the blade row. A derivation similar to that above nives:

$$
\omega_{2}^{2}-\varphi_{2}^{2}\left(1-\frac{\omega^{2}}{2 C_{p} T_{2}^{\top 1}}\right)^{\frac{-2}{\gamma-I}}=0
$$

where

$$
p_{2}=\frac{i n}{\rho_{2} r_{2} n_{2}} \frac{\text { cos } a_{2}}{\cos }
$$

which is solved at each grid point within the blade row. Once the flcw conditions are known at the trailing eage, Eq. (20) can be used for the downstream region.

\section{Boundary Conditions}

At the inlet, total pressure, total temperature, and whirl rva are specified. For suosunic inflow the governing equations have one negative Eigenvalue so that one variable at the inlet must De computed as part of the solution. Here a characteristic relation is used to extrapolate the upstrean-running Riemann Invariant to the inlet.
The axisymetric m-momentum equation may be written as:

$\partial t^{R^{-}}+\left(v_{m}-a\right) z_{m} R^{-}=\left(v_{\theta}^{2}+a v_{m}\right) r_{m} / r+a v_{m} h_{m} / h$

where

$$
R^{-}=v_{m}-\frac{2 d}{r-I}
$$

is the upstream-running Rieniann invariant. For steady axisymetric flow Eq. (22) may be written ds:

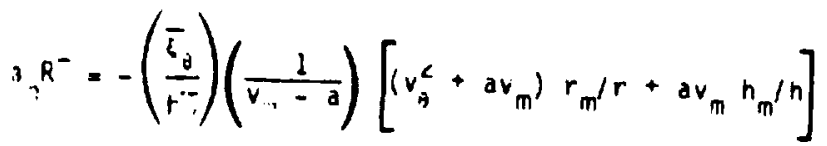

Equation (23) is backward-differenced and solved for $R^{-}$at the inlet. Then $v_{m}$ is found froin $R^{-}$and the specified whirl $r v_{\theta}$ and total temperature $T$ ' using:

$v_{m}=$

$$
\frac{(r-1) R^{-}+\sqrt{\left.(r+1)\left(4 C_{p^{\prime}}{ }^{\prime}-2 v_{\theta}^{2}\right)-2 ! r-1\right)\left(R^{-}\right)^{2}}}{(r+T)}
$$

Density ano energy are found using isentropic relat ons.

At the exit the static pressure is specified and the other flow quantities are found using first-order extrapolation. Zeroth-order extrapolation is not usually sufficient in cylindrical cuoroinate systems because the radius and thus the velocity may change between grid noints. For the same reason the inlet and exit boundaries cannot be pliced arbitrarily far from the blades. Too small a radius can cause tile flow to be supersonic and to large a radius cal cause the velccity to approach zero at the boundaries.

Blade surface pressurt's are found from the normal momentum equation:

$$
\begin{aligned}
& \left(\varepsilon_{m} n_{m}+\bar{\xi}_{\theta} \bar{n}_{\theta}\right) a_{\varepsilon} p+\left(n_{m}^{2}+\bar{n}_{\theta}^{2} ; \partial_{n} p\right. \\
& =-\rho w^{2}\left(n_{m} \partial_{\varepsilon} v_{m}+\bar{n}_{\theta} \partial_{\varepsilon} v_{\theta}\right)+D_{\theta \theta}\left(n_{m} v_{\theta}-\bar{n}_{\theta} v_{m}\right) r_{m} / r
\end{aligned}
$$

where $w^{k}=0$ on the surface for viscous flows. points.

Periodic boundaries are sulved like interior
s.

\section{Fine-Gria Algorithm}

On the fine grid an explicit two-stage finite-difference Rynge-kutta algorithm based on the work of Jameson 15 is used. It is given be low as applied to EQ. (7). 


$$
D=C\left(w_{2} 1 D_{56} q_{\xi 6}-H_{4} q_{565 \zeta}\right)
$$

$$
\begin{aligned}
& \hat{q}^{(1)}=\hat{q}^{n}-a_{1} \Delta t R^{n} \\
& \hat{q}^{(2)}=\hat{q}^{n}-\Delta t R^{(1)}
\end{aligned}
$$

where

$$
\begin{aligned}
& a_{1}=1.2 \\
& R_{i, j}=-\hat{k}_{1, j}+\frac{1}{2}\left[\left(\hat{F}_{i+1, j}-\hat{F}_{i-1, j}\right)\right. \\
&\left.+\left(\hat{G}_{i, j+1}-\hat{G}_{i, j-1}\right)-\operatorname{Re}^{-1}\left(\hat{S}_{i, j+1}-\hat{s}_{i, j-1}\right)\right]
\end{aligned}
$$

Artificial dissipatior:

$$
\hat{q}^{n+1}=\hat{q}^{(2)}+o^{(2)}
$$

\section{Collect residuals}

$$
\Delta \hat{q}^{n+1}=\hat{q}^{n+1}-\hat{q}^{n}
$$

The two-stage scheme given by Eq. (26) has a Courant number limit of one. It is used in preference to a niaher-order scheme with a higher Courant number limit because the multigrio scheme used here also has a Courant number limit of one.

four minor advantages of the Runge-kutta scheme over the MacCormack scheme are noted:

1. A steady Runge-Kutta solution is independent of the time step while a steady Maccormack solution is not. This is not true when the artificial dissipation is addes in a fractional step as in EQ. (27).

\section{The centrally-differenced Runge-kutta} scheme produces perfectly symmetric solutions for symmetric prorlems while the one-sideo MacCormack scheme does not.

3. For a two-dimensional problem with centrally-differenced metrics, the Runge-kutta scheme exactly conserves a iree stream while the MacCormack scheme does not. Although the PungeKutta scheme is not fully consorvative for the quasi-three-dimensional problem because of the source term, it has been found to possess better conservation properties in general.

4. The Runge-Kutta scheme is slightly eacisi to program than the MacCormack scheme.

Differences in convergence rates betwoinn the two schemes are negligiole for Courant num.jers near one.

\section{Artificial Dissipation}

Dissipative terms consisting of second and fourth differences are added to prevent odd-even point decoupling and to llow shock capturing. The dissipative terms are similar to chose used by Jameson 15 and others. A one-dimensiona? version (6-direction) is given below. In two dimensions the dissipation is applied as a sequence of one-dimensional operators. where

$$
\begin{aligned}
& c=\frac{\Delta t}{j} \sqrt{n_{m}^{2}+n_{\theta}^{-2}} \\
& u_{2}=0(1) \\
& \nu_{4}=0(1 / 26)
\end{aligned}
$$

The terms in the coefficient $c$ balance similar terms in Eq. (26). In smooth regiors of the flow the dissipative terms are of third order and tnus do not detract from the formal secondorder accuracy of the fine-grid scheme. In regions of the flow where the secono difference of the pressure is large, the second-difference dissipation becomes locally ce first order. Note that in other work including Ref. 15 the term 'PG' is commonly divided by an average pressure. Th $\{\xi$ is not done here because pressures through centrifiugal compressor can increase ty factors on the ordigr of five, which woulc decrease the dissipation correspondingly.

\section{Stability Analysis}

h stadility andysis of the fine-grid algorithin is performed in two parts. The first cart exarilnes the mooel problem considered by Jameson 15 and is used nere to choose tre parameters in tre two-stage jcheme. The model problem is the oneaimensional convection equation with thira-order artificial oissipation:

$$
q_{t}+q_{x}+\mu \Delta x^{3} r_{x \times x x}=0
$$

Applying the two stage schene (Eqs. (26) and (27); gives:

$$
\begin{aligned}
& q^{(1)}=q^{n}-a_{1} \frac{1}{2}\left(a_{j+1}-q_{j-1}\right)^{n} \\
& q^{(2)}=q^{n}-\frac{1}{2}\left(a_{j+1}-a_{j-1}\right)(1) \\
& q^{n+1}=q^{(2)}-4 \lambda\left(a_{j-2}-4 a_{j-1}+6 a_{j} \cdot 4 q_{j+1}+a_{j+2}\right) \\
& \text { where } A=\Delta t / \Delta x \text { is the Coui ant number. }
\end{aligned}
$$
solution

$$
\text { If we consider a fourier comporient of the }
$$

$$
q_{j}^{n}=v^{n} e^{i p x}=v^{n} e^{i j \xi}
$$

wrere $V$ is the amplitiode, $P$ is the wave number. $\xi=p \Delta x$, and $i=\sqrt{-1}$, then the amplification factor is given by:

$$
\begin{aligned}
g=\frac{v^{n+1}}{v^{n}} & =\left[1-a_{4 \lambda}(1-\cos 6)^{2}\right] \\
& \times\left(1-\lambda i \sin \varepsilon-a_{1} \lambda^{2} \sin ^{2} \xi\right) \\
& =\text { damping factor } x \text { characteristic } \\
& \text { rolynomial for unoamped scheme. }
\end{aligned}
$$


The damping coefficient $\psi$ is chosen such that $g=0$ d $f=x$, yiving $w-1 /(1, \lambda)$.

An undamped n-stage scheme can only be stable to $\lambda=n-1$. $50 \lambda$ is taken to be one while at is chosen. Figure 2 shows a plot of Eq. $(33)$ for several values of al, with $\lambda=1$ and $\mu=1 /(16 \lambda)$. It can be seen that the twostage screme is stable for $0.5<a 1 \leq 1.7$, with a $\approx 1.2$ giving the best damping over the range of frequencies. For $1-1.2$ and $\mu-1 /(16 \lambda)$ it can be shown that the two-stage scheme is stable for Courant numb ars $i \leq 1.1$. In general the twn-stage scheme is first-s-der accurate in time. It is second-order accurate in time only if $a_{1}=0.5$.

The second part of the stability and lysts considers the linearized Euler subset of the governi..g equations (Eqs. (7) ano (8)). A Von Neumann analysis shows the stability linit on tile lime step to be:

$$
\Delta t \leq\left[\begin{array}{c}
w^{*}+w^{n}+a \sqrt{\left(\varepsilon_{m}+n_{m}\right)^{2}+\left(\bar{s}_{\theta}+\bar{n}_{p}\right)^{2}} \\
\left.+\frac{v_{\theta} r_{m} / r}{2 a \sqrt{\left(\varepsilon_{m}+n_{m}\right)^{2}+\left(\bar{s}_{\theta}+\bar{n}_{a}\right)^{2}}}\right]
\end{array}\right]^{-1}
$$

which is implemented as:

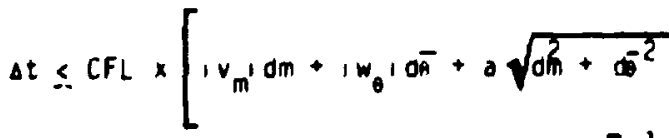

$$
\begin{aligned}
& \left.+\frac{v_{\theta} r_{m} d r}{2 a \sqrt{d m^{2}+\sigma^{-2}}}\right]^{-1}
\end{aligned}
$$

where

$$
\begin{aligned}
& d m^{2}=16 m^{1}+1 n_{m} \\
& d \theta^{2}=1 \overline{6}_{0}+1 \bar{n}_{0}
\end{aligned}
$$

A spatially-variable time-step at $j$ is used in the two-stage scheme (Eqs. (26) dho (27)) to accelerate convergence to steady state. Equation (34) is used to choose the time step at each grid point such that the Courant number is constant, typic.lly CFL $=0.95$. Time steps are calculated based on the initial conditions. They are stored and not updated ouring the calculations.

\section{Multigrid Algorithm}

The multigrid algorithm orig/nated oy $\mathrm{Ni}^{16}$ and modified by Johnsun and Chimal -19 is used to accelerate convergence of the fine-grid algorithm. $\mathrm{Mi}$ 's scheme is basically a one-step Lax-Wendroff schem: applies on a coarse grid. Ni used it to accelerate his own fine-grid Euler scheme. 16 Johnson adapted $N$ !'s method to other f $\{$ ge-grid schemes including MacCormack's scheme. 19 He also used it for viscous flows by demonstrating that dissipative terms need nou be included on the coarse grids. Thus the multigrid scheme used here is based solely on the Euler equat ton:. It is eitirely indeperdent of the viscous terms, the turbin'ence model, and the artificial dissipation used on the fine grid.

One-step Lax-Hendroff sc' nes including $N I^{\prime}$ 's scheme require temporal derivatives of the flux vectors. These terms are computed as the Jacobian inatrix of the flux vector times the temporal difference of the solution vector. Johnson replaced these lengthy computations with a direct temporal difference of the flux vector using the old and new solutions on the fine grid. 18 This "flux based" scheme is consideriuly simpler than Ni's original scheme.

The flux-based multigrid scheme is derived by expanding the flite-grid change $\Delta q^{n+1}$, (Éq. (28)), in a Taylor series.

$$
\Delta \hat{q}^{n+2}=\Delta \hat{a}^{n+1}+\Delta t\left(\Delta \hat{q}^{n+1}\right) t+0\left(\Delta t^{2}\right)
$$

The Euler equations are used to replace the third ierm.

$$
\left(\Delta \hat{q}^{n+1}\right)_{t}=\left[\Delta t\left(\hat{K}-\hat{F}_{\xi}-\hat{G}_{n}\right)\right]_{t}
$$

Interchanging the space and time derivatives and using backwaro oifferencing in time gives:

$$
\begin{aligned}
\left(\Delta \hat{q}^{n+1}\right)_{t} & =\Delta t\left(\hat{k}_{t}-\hat{F}_{t}-\hat{G}_{t n}\right) \\
& =\left(\hat{k}^{n+1}-\hat{k}^{n}\right)-\left(\hat{F}^{n+1}-\hat{F}^{n}\right)_{t}-\left(\hat{G}^{n+1}-\hat{G}^{n}\right)_{n}
\end{aligned}
$$

and finally

$\Delta \hat{q}^{n+2}=\Delta \hat{q}^{n+1}+\Delta t\left[\Delta \hat{F}-(\Delta \hat{F})_{\varepsilon}-(\Delta \vec{G})_{n}\right]^{n+1}$

where

$$
\Delta \hat{K}^{n+1}=\hat{K}\left(q^{n+1}\right)-\hat{K}\left(q^{n}\right) \text {, etc. }
$$

Equation (36) is implemented on a coarse grid with spacing $l \alpha$, and $l \Delta \eta$, and time step $\sim \Delta t_{i, j}, l=2,4,8, \ldots$. , tising:

$$
\begin{aligned}
\Delta \hat{q}^{n+2}=\frac{1}{4} & \left\{\left[\Delta \hat{q}+\Delta t_{i, j}(l \Delta \hat{K}-\Delta \hat{F}-\Delta \hat{G})\right]_{i+l, j+l}\right. \\
& +\left[\Delta \hat{q}+\Delta t_{i, j}(2 \Delta \hat{K}-\Delta \hat{F}+\Delta \hat{G})\right]_{i+l, j-l} \\
& +\left[\Delta \hat{q}+\Delta t_{i, j}(l \Delta \hat{K}+\Delta \hat{F}-\Delta \hat{G})\right]_{i-l, j+l} \\
& \left.+\left[\Delta \hat{q}+\Delta t_{i, j}(l \Delta \hat{K}+\Delta \hat{F}+\Delta \hat{G})\right]_{i-l, j-l}\right\} n+1
\end{aligned}
$$

Fine-grid changes $\Delta \hat{q}^{n+l}$ are restricted to the coarse orid by injection. The multigrid algorithm (Eq. (37)) computes coarse-grid corrections $\Delta a^{n+2}$ based on changes in the fine-grid solution. Thus the coarse-grid corrections must vanish if the fine-grid solution converges, thereby retaining fine-grid accuracy. 
The coarse-grid corrections are prolonged back to the fine grit using bilinear interpolation and the fine-grid solution is updated. The process may then be repeated on a coarser grid.

\section{Vectorization}

The explicit Ruige-Kutta and multigrid aigorithns used here have been highly vectorized for the Cray I-S at NASA Lewis Research Center. Indeed the Runge-Kutta computations were clocked at about $40 \mathrm{milli}$ ion floating point operations/sec ( $40 \mathrm{mflops}$ ) for an Euler solution on a 113 by 25 grid. The efficiancy of the multigrid computation: decreases as the grid gets coarser and the vectors get shorter.

The code was redimelisioned for each grid size run and required $260 \mathrm{~K}$ words of memory fur the largest grid (161 by 33$)$. The quasi-threeaimensional cooe requires about 20 perçent mor? storage than the two-dimensional code.

\section{Resuits}

Results are presented for the following problems: a cascade of thin flat plates with round leading edges, a centrifugal impeller, and a vaned-radial diffuser. Both Euler and NavierStokes results are presented.

To aid in developing the quasi-threedimensional code and to illustrate the capabilities of the analysis, a model problem was developed representing a cascade of thin flat plates with round leading adges. The plate has unit chord, four percent thickness, and pitch of 0.7 . Figure 3 shows the computaticral grid around the plate. The inviscid flow grid had 113 by 25 points with $5^{\circ}$ spacing around the leading edge circle and a normal spacing of $4.8 \times 10^{-4}$. The viscous flow grid had 113 by 33 points with. normal spacing of $5 \times 10^{-5}$. The grid shown in Fig. 3, and most of the subsequent grid and contour plots in this paper, are drawn in a t.ansformed plane in which the abscissa is $f \mathrm{dm} / r$ and the ordinate is $\theta$. This transformation preserves angles and is discussed in Ref. 10.

Grids are generated in an $(m, \bar{r})$ coordinate systen where $\bar{r}$ is some mean rarius, and are independent of the local radius $r$ and streamsurface thickness $h$. Values of $r$ and $h$ are supp:led to the quasi-three-dimensional code later and can be varied to simulate different geometries.

A flat cascade was simulated by setting $r$ - constant and $h=$ constent. Figure 4 shows static pressure contours for an Euler solution with an inlet Mach number of 0.33 . (Titles on the figures give nominal valuer. of Mach number, Reynolos number, and flow angle based on the leading-eoge velocity triangles given as input. Actual inlet conditions may change as the solution develops.) The contours shor: the stagnation region and a milo acceleration due to blockage. ldentical results were obtained using the twooinensional code. 6 The solution was run 5000 cycles with two multigrid levels and took $165 \mathrm{sec}$ on the Cray. The residuals were reduced eight decades so the solution could have been stopped at about 2000 cycles with a three-decade reduction in the resiouals.
A radial duct with constant crcss-sectiunal arta was simulated by setting $r=m$ and $r \times h$ - constant. The solution is identical to the flat cascade results shown in $\mathrm{Fig}$. 4 .

A radial diffuser with 36 blades was simulated by setting $r=m$ and $h=$ constant. Figure 5 shows static pressure contours for an Euler solution with an inlet Mach nimber of 0.38 and an exit Mach number of 0.27 . The plot is shown in polar coordinates. The contours show the diffusion of the flo's due to the area change. The solution was run 2500 cycles with two multigrid levels and took $92 \mathrm{sec}$ on the Cray. The residuals were reduced 6 decades so the solution could have been stopped at about 1250 cycles with a threedecave reduction in the resiauals.

A centrifugal impeller with 36 blades was simulated by setting $r=m, n=$ constant, and a rotation rate of $1000 \mathrm{rad} / \mathrm{sec}(9550 \mathrm{rpm})$. Figure 6 shows Mach number contours for a turoulent solution with an inlet Mach number of 0.5 and an exit Mach number of 0.41 . The impeller produces a total pressure ratio of 1.05 . The plot is stown in polar coordinates with rotation upwaros. The inlet whirl is zero but becaust of the rotation the in et relative flow angle is $10.6^{\circ}$. Tris incidence desymmetrizes the flow and pruduces pressure loading on the bladt. At the exit this loading conflicts with the specified exit pressure and is responsible for the kinks in the contours there.

Figure 7 shows convergence histories for the previous example with various levels of multigrid. Convergenca is taken to be a three decade drop in the maximum residual to $2 \times 10^{-5}$. For this example iwo grids are 1.64 times faster than the fine grid and three grias are 3.12 times faster than the fine grid. CPU timas are included on the figure.

The remaining results are for a nominally $6: 1$ total-pressure ratio centrifugal impeller and matching vaned-radial diffuser. These components were designed by the Air Force for use in an auxilliary jower unit and were modified and tested at NASA Lew is Research Center. The compressor has a diameter of $16.1 \mathrm{~cm}$. It has 19 blades and was desinned for a total pressure ratio of 5.9:1 with d mass flow of $1.033 \mathrm{~kg} / \mathrm{sec}$ at $68384 \mathrm{rpm}$. The diffuser has 27 blades and diameter of $25.1 \mathrm{~cm}$. Further details concerning tne components can de be found in Ref. 25.

Figure 8 shows the computational grid used for the impeller. The grid has 161 by 33 points $(16)$ by 17 shown) with a normal spacing of $3 \times 10^{-4} \mathrm{~cm}$ for inviscid flows and $5 \times 10^{-5} \mathrm{~cm}$ for viscous flows. The leading edge is round with a radius of $0.045 \mathrm{~cm}$ and the grid points are $7^{\circ}$ apart.

Figure 9 shows normalizeo radius (RMSP $=r$ ). stream-surface thickness (BESP $=h$ ), and their product $(R \star B=r h)$ versus fracticn of impeller chord. These values were taken from a meridional analysis.

Euler and Navier-Stokes solutions were each run 2000 cycles with two multigrid levels, reducing the maximum residual three decades. The Euler solution took $127 \mathrm{sec}$ anj the Navier-Stokes solution took $185 \mathrm{sec}$ on the cray. 
Surface static pressure distributions for the impeller are compared in Fig. 10. Three solutions with identical mass flows are shown: an Euler solution (dashed), Navier-Stokes solution (solid), and a panel solution (circles, 10 ). The Euler and Navier-Stokes solutions were run with various exit pressures until the desirtd mass flow was obtained. The shapes of the three pressure distributions are similar but the panel solution has higher pressure levels since it is loss-free. The Euler solution has strong shock losses whtch lower the pressure levels. The Mavier-Stokes soluition has weaker shocks due to viscous smoothing of the leading edge, but blockage eff 'tts decrease the pressure levels overall.

F igure 11 shows relative Mach number contours for the Esler solution. The dashed line is the sonic line. At the inlet the relative Mach number is 0.91 . At the leading edge the flow has $10^{\circ}$ of iricidence. This produces a large supersonic bubble with a peak Mach number of 1.92 on the suction (upper) surface. The bubble terminates with a normal shock that is smeared due to grid shearing in this region. There is also a tiny supersonic bubble on the pressure (lower) surface which is not visible at this scale.

Some interesting flow phenomena evident in Fig. Il can be explained by the concept of a relative eddy. The flow through an impelier is predominantly inviscid and tends to remain irrotational. The blade row in Fig. 11 is rotating downward and so adds clockivise vorticity to the flow. To remain irrotational the flow develops a counterclnckwise circulation within the passage. Thus the flow can be modelled as a superposition of a through-f low component and a component rotating counter to the olade row called a relat: ie eddy. The effect of the relative eddy is to accelerate the flow on the suction surface and decelerate the flow on the pressure surface as can be seen in Fig. 11 .

A more aramatic effect of the relative eday is to sweep the unconfined flow beyond the tratiing eoge up and away from the blade in a spiral. with slip line leaving the trailing edge. As a comon example of slip from rotating machinery, consider the flow of water from a lawn spririxier as viewed rotating with the sprinkler. The llow spirals up and opposite to the rotation of che sprinkler. It is emphasized that this is strictly an inviscid phenomena.

Figure 12 shows relative Mach nimber contours for a Navier-Stokes solut ton for the impeller. Here viscous effects reduce the peak suctionsurface Mach number to 1.72 and the peak pressuresurface Mach number to just over 1.0. Both surfares have small leading-edge sepalation bubbles that are barely resolved on this grid. The pressure surface quickly develops a thick boundary 1 ayer and the suction-surface boundary layer thiskens after the shock. This blockage causes the viscous pressure levels to be lower than the inviscio ?evels in Fig. 10 . The rotation energizes and diminishes the boundary layers on thr. radial portions of the blade.

Here again the relative eody sweeps the flow of the trailing eoge in a spiral. Jince the flow coes not follow the grid lines the thin-layer assumption may be invalid and diffusion across the wake may not be properly accounted for. This is one shortcoming of the present analysis. However, since the irailing-edge slip is an inviscid phenor :na it is felt that the character of the solution is correct.

F igure 13 shows the computational grid for the radial diffuser vane. The grid has 145 by 33 points (145 by 17 shown) with a n-:mal spacing of $2.5 \times 10^{-4} \mathrm{~cm}$ for inviscla flows and $6 \times 10^{-5} \mathrm{~cm}$ for aiscous flows. The round leading edge has a radius of $0.025 \mathrm{~cm}$ and the grid points are $7^{\circ}$ apart. At the trailing edge the actual vane is cut off at constant $r$ adius and the duct turns axially. For this analysis the trailing eoge was sharpened and the duct was extended radially.

Figure 14 showr the normalizeo radius and strearl-surface thickness versus fraction of vane chord. These values were taken from combined merioional/boundary-layer analys is.8,9 Aitinough the vanes have constant height, boundary layer blockage decreases the flow area by nearly 50 percelıt.

An Euler solution was run 4000 cycles with two multigrio levels, taking $214 \mathrm{sec}$ on the Cray. A Navier-Stokes solution was run 2000 cycles with three multigrid levels, taking $179 \mathrm{sec}$. In each case the maximum residual was reduced three decades.

Surface static pressure distributions for the oiffuser vane are compared in Fig. 15. Again. panel. Euler, and Navier-Stokes solutions are compared. The shapes of the pressure distributions are similar but the panel solution has higher pressure levels since it is loss-free. The Euler and iavier-Stokes solutions each have small supersonic bubbles terminated by normal shocks near the leading edge, and the losses lower the pressure levels. Additional olockage effects cause the viscous pressure levels to be even lower than the inviscto levels.

F igure 16 shows Mach number cuntours for the Euler solution. The inlet Mach number is exactly 1.0 out the radial component is only 0.29 so the flow is subsonic in character. Because of the increasing radius the total Mach number drops to about 0.83 near the leading edge. The flow has a slight positive incidence at the leading edge and the flow accelerates to a pesk Mach number of about 1.5 on the suction surfa:e. The tiny supersonic bubble (visible only as a black dot at this scale) terminates with shock at about 0.26 percent chord. The pressure surface develops an even smalier supersonic bubble. The diffusion through the passage is evident in fig. 16.

F igure 17 shows Mach number contours for the Navier-Stokes solution. Here viswous effects at the leading edge entire'y suppress the formation of iupersonic bubbles. The thick boundary layers that ouvelop in the diffuser are obvious, but the flce remains attached on both surfaces.

\section{Concluaing Remarks}

A quasi-three-oimensional Euler ano NavierStokes anal $:$ is technique has been developed for Dlade-to-bidoe flows in turbomachinery. The enialysis solves the :hin-layer Navier-stokes 
equations written ir. general coordinates for an axisymmetric stream surface, and accounts for the effects of blade-row rotation, radius change, and stream-iurface thickness. It is believed that this is the first Navier-Stokes arialysis to include these effects.

The solution technique is a two-stage RungeKutta scheme based in the work of Jameson. Efficlency is achieved throigh use of vectorization. a spat:ally-variable time-step, and a multigrid scheme based on Johnson's revisions of NI's scheme. Thi multigrid scheme typically reduces the CPU time required by the fine grid scheme alonp by a tactor of about three, for both inviscid and viscous irows.

Results for a model problem show the analysis to be viable for a variety of axial, radial, and rotating geometries. Results for a centrifugal impeller and a radial diffuser vane show that the analysis can predict a number of phenomena that are not accounted for in previous analyses. These phenomena include: leadino-edge stagnaticn points, leading-edge separation, supersonic regions and shocks, blade-surface boundary layer growth, and trailing-edge slip lines.

It is thought that the ability to predict these phenomena rapidly for general geometries could make tha quasi-three-dimensional andysis a useful tool for turhomachinery design. Furthermoie the quasi-three-dimensional analys is can provide insight into both physical and numerical problems that can be expected with fully threeaimensional problems in the future.

\section{References}

1. McFarland, E.R., "Solution of Plane Cascade Flow UEing Improved Surface Singularity Methods," Journal of Engineering for Power. Vol. 104, No. 3, JuTy 1982, PD. 668-674.

2. Ives, D.C., an.s Liutermo 2 A, J.F., "SecondOrder-Accurate Calculation of iransonic flow over Turbomechinery Cascadas," AIAA Journal. Vol. 17. No. 8, Aug. 1979, p. 870-876.

3. Denton, J.0., "An Improvec Time Marching Method for Ti:rbomachinery F low Calculation," Journal of Engineering for Power, Vol. 105, No. 3, Juty Iy83, pi. 514-5.34.

4. Subramanian, S.V., and Bozzola, R., "Afplica-tion of Runge Kutta Time Marching Schene for the Computation of Transonic $F$ lows in Turbo-- machines, "A.,A Paper 85-13:2, July 1985 .

5. Stegel, J.L., Pulliam, T.H., and Chima, R.V., "An Imo icicit Finite-Difference Code for Invisc and viscous Caccade Flow, "A!AA Paper 80-:4 7 , July 1980 .

6. Chima, R.V., "Analysis of Inviscid and Viscous Flows in Cascades with an Explicit MultiplfGrid Algorithm," AIAA Paper 84-1663. June 1984 .
7. Wu, C.-H., "A General Theory of ThreeDimensicnal Flow in Subsonic and Supersonic Turboriochines of Axial-, Radial-, and MixedFlow "ypes," NACA TN-2604, 1952.

B. Katsanic, T., and McNally, W.D., "Revised FORTRAN Program for Calculating Velocities . nd Streamlines on the Hub-Shroud Midchannel Stream Surtace of an Axial-, Radial-, or Mixed-Flow Turbomachine or Annular Duct, I User's Manual," MASA TN-0-8430, 1977.

9. McNally, W.D., "FORTRAN Program for Calculating Comprejsible Laminar and Turbulent Boundary Layers in Arbitrary Pressure Gradients." NASA TN-D. $: € 81,1970$.

10. McFarland, E.R., "A Rapid Blade-to-Blade Solution for lise in Turbomachinery Jesign," Journal of Engineering for Gas Turbines and Powfr, Vol. 106, Mo. ?, Apr. 1984, pp. $376-382$

11. Katsanis, T., "FORTRAN Procirani iar Calculating Transonic Velocities on a Flade-to-Blade Stream Surface of a Turbomachine," NASA TN- $[-5427,1969$.

12. Farrel1, C., and Adamczyk, J., "Full Potential Solution of Transoric Quasi-.j-D F low Through a Cascade Using Artificial Compressibility," Journal of Engineering for Power, Vol. 104, No. 1, Jan. 198?. OD. $143-153$.

13. Bertheau, F., Ribaud, Y., end Millour, V., "Transonic Blade-tu-Blade Calculations in an Axial, Radial, or Mixed Flcw Cascade Equipped with Splitter Blodes, "ASME Paper 85-GT-86, Mar. 1985.

14. MacCormack, R.W. "The Effect of Viscosity in Hypervelocity Impact. Cratering," AIAA Paper 69-354, Apr. 1969.

15. Jameson, A., and Baker, T.J., "Multigrid Solution of the Euler Ejuations for Aircraft Configurations," AIAA Paper 84-0093, Jan. 1384 .

16. Ni, R.H., "A Multiple Grid Scheme for Solving the Euler Equations, "AlAA Paper 81-1025, A:AA 5 th Computational Fluid Dynamics conference, June :981, pp, 257-264.

17. Johnson, F.M., "Convergencs Accele:-at un of Hiscous Fluw Computations,' NASA TM-83039, 1982 .

i8. Johnson, G.M., "Fll.x-Based Acceleration of the Euler Equations," NASA TA-83453, 1983.

19. Chima, R.V., anj iuhnson, G.M., "Efiticie.st Solution of the Euler and Navier-Stckes Equations with a Vectorized Multiple-Grid Algorithm," AlAA Journal, Voi. 23, No. 1 . Jan. 1985, pp. 23-32.

20. Katsanis, T., "fipplications of the contravariant Form of the Navier-Stokes Equations," HIASA TM-83448, 1983. 
21. Baldwin. B.S., and Lomax, H., "Thtn-Layer Approximation and Algebraic Model for SeDarated Turbulerit Flows," AIAA Paper $7 \mathcal{C}-257$, Jan. 1978.

22. Sorenson, R.L., "A Computer Program to Generate Two-Dimensional Grids About Alrfol's and Other Shapes by the Use of Poisson's Equation." MASA TM-81198, 1980.

23. Steger, J.L., and Sorenson, R.L., Mutomatic Mesh-Point Clustering Mear a Boundary in Grid Generation with Elliptic Partial Differential Equations." Journal of Computetional Physics, Vot. 33, No. 3, Dec. I979, 0. 405-4to.
24. Barton, J.T., and Pulliam, T.H., "Airfoil Computation at High Angles of Attack. In.iscid and Viscous Phenomena," AIAA Paper 34-0524, Jan. 1984.

25. Klassen, H.A., Wood, J.R., and Schumann, L.F. "Experimental Performance of a 16.10-Cent imeter-Tip-Dianeter Sweptbark Centrifugal Compressor Designed for a $6: 1$ Pressure Ratio," NASA TM X-3552, 1977. 


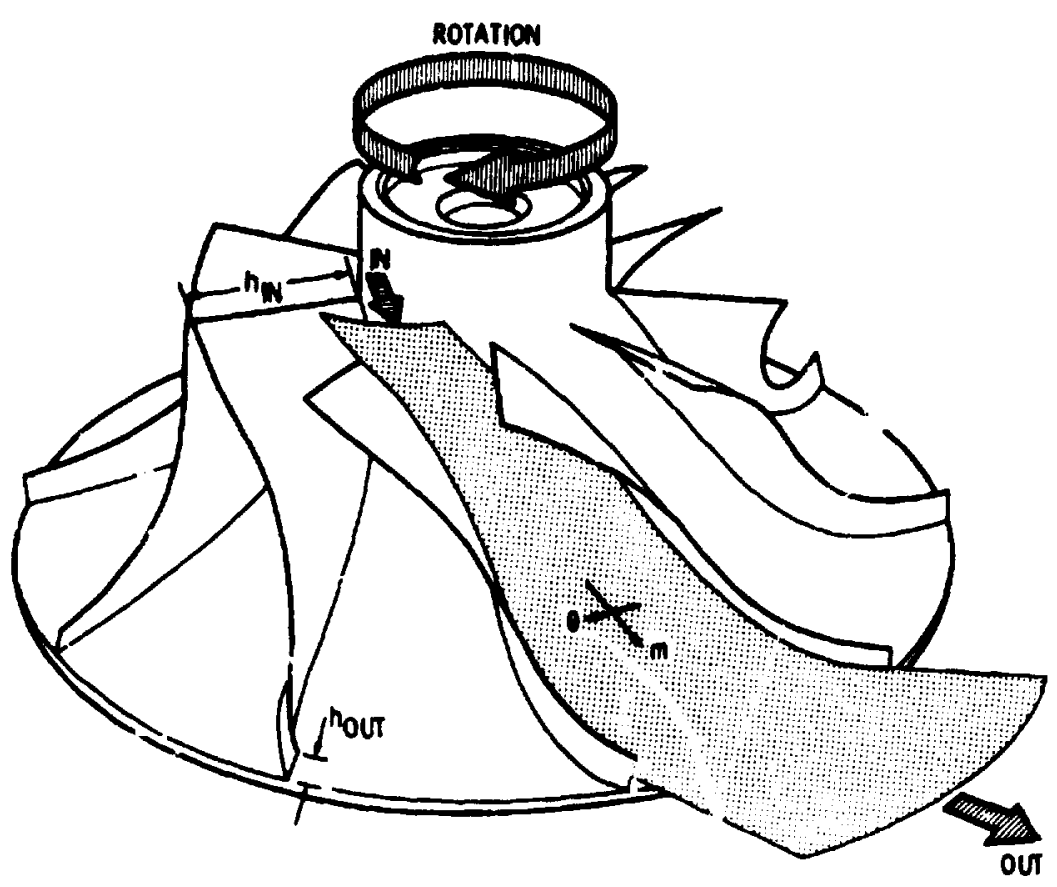

Figure 1. - Ouasi-three-dimer sional streali: surl.ice and coordinate system for a centrifugal con.,ressor.

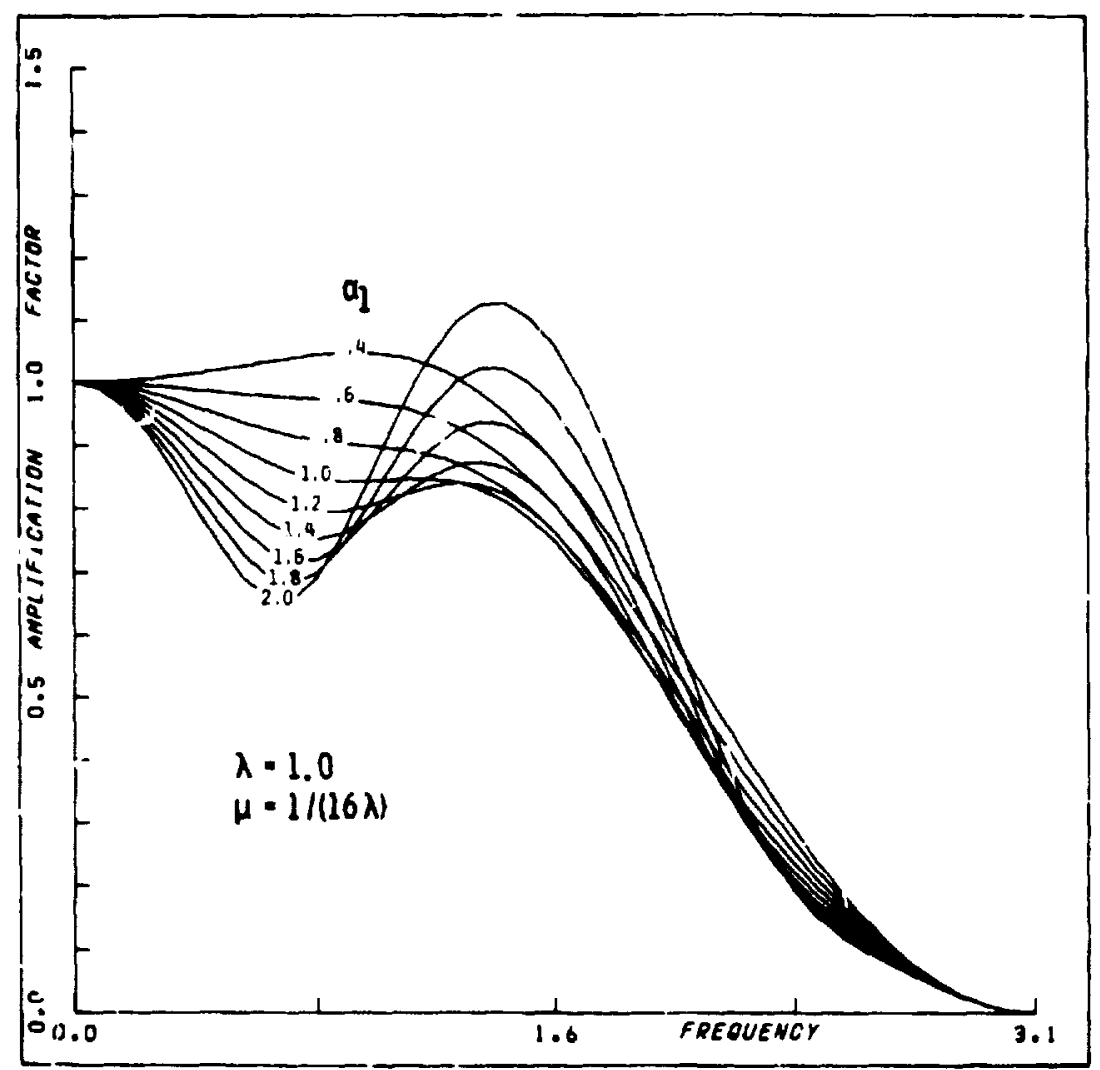

Figure 2. - Amplirication factor for two-stage Runge-Kutta scheine. 


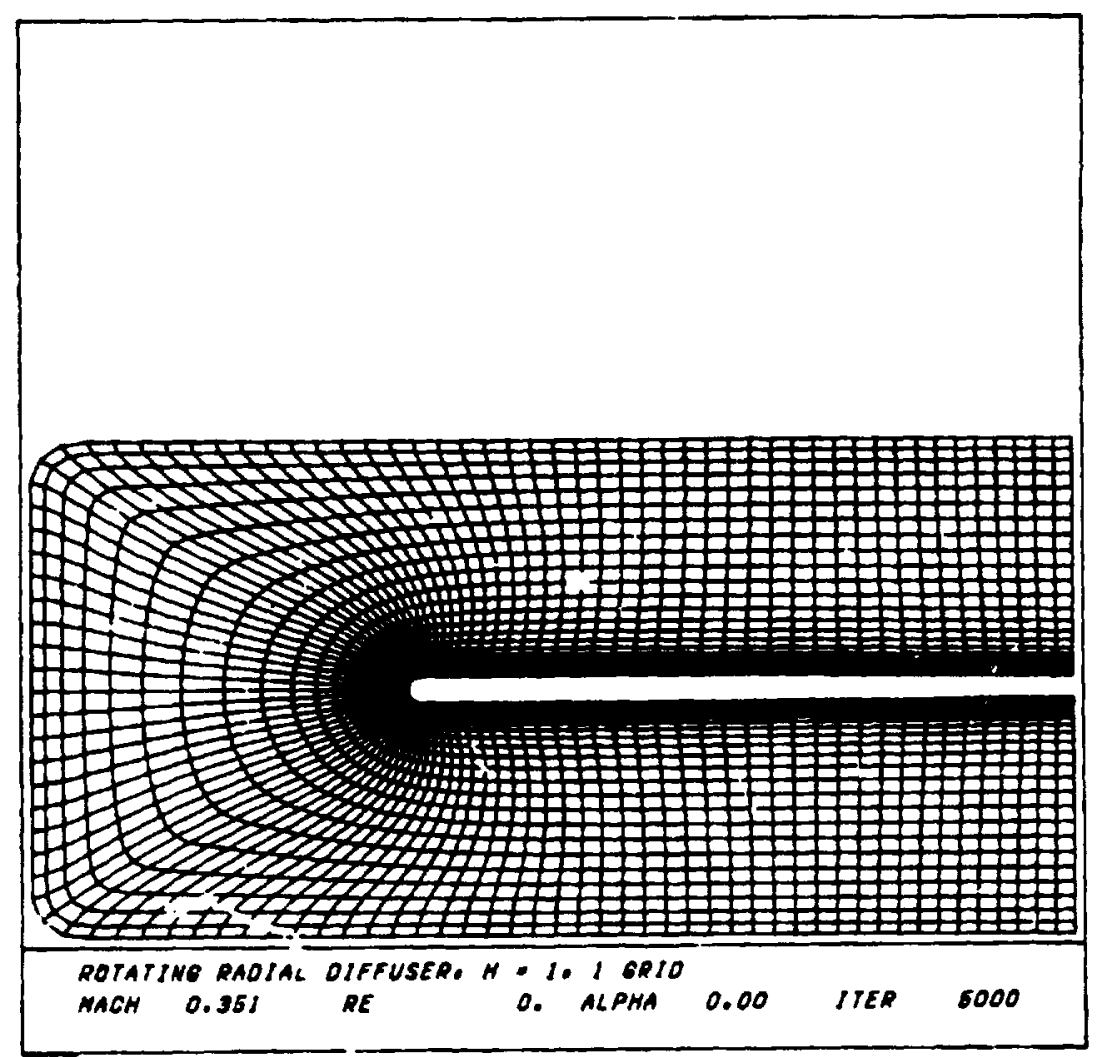

Figure 3. - Computational grid tor flat plate model problem.

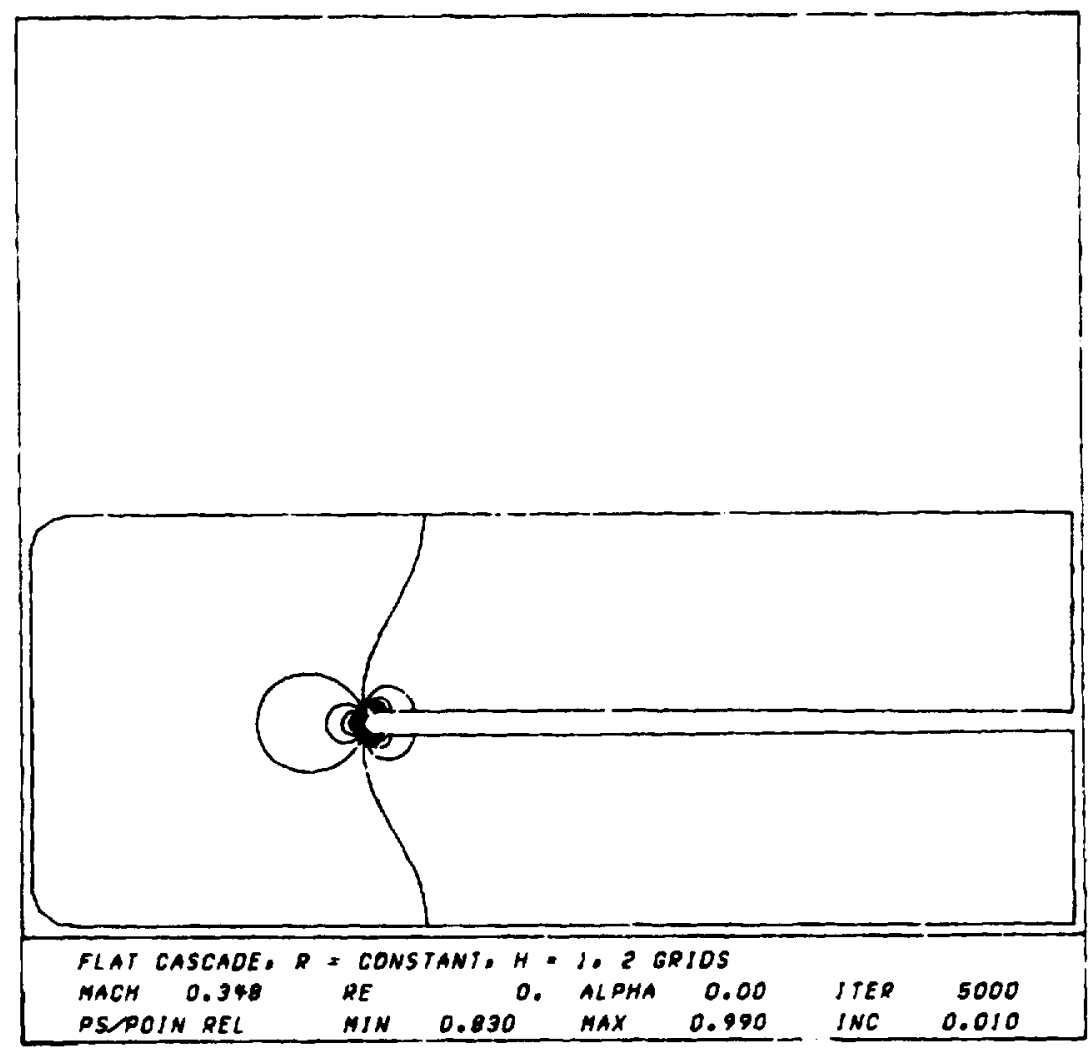

Figure 4. - Static pressure contours for inviscid flat plate cascade model. 


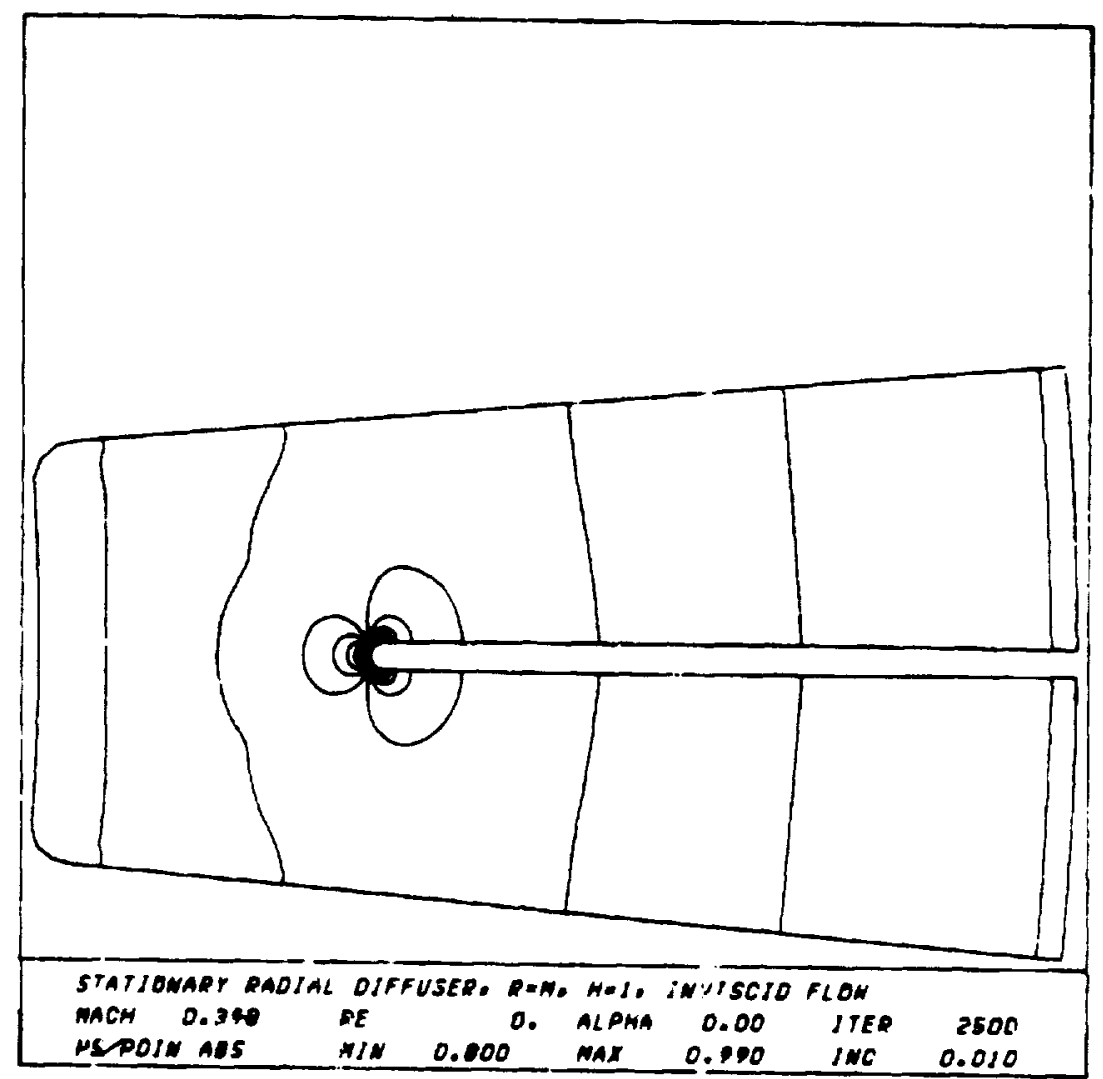

Figure 5. - Static pressure contours for invischi redial diffuser model.

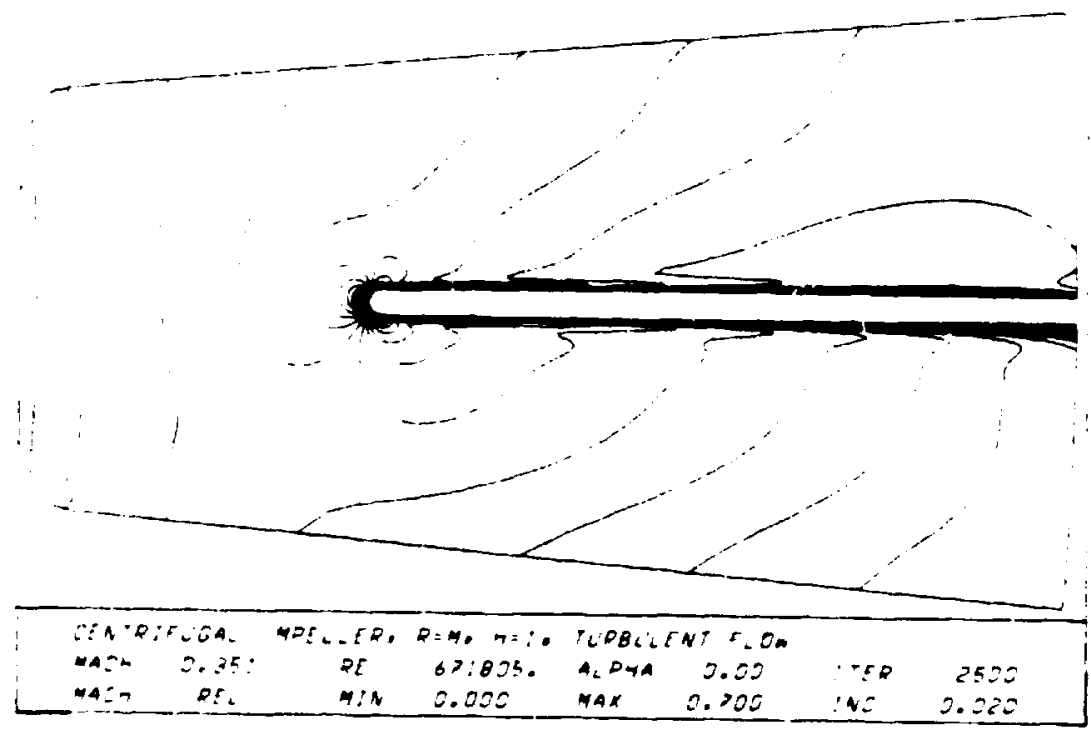

Figure 6. - Mach number contours for turbulent centrifugal impeller model. 


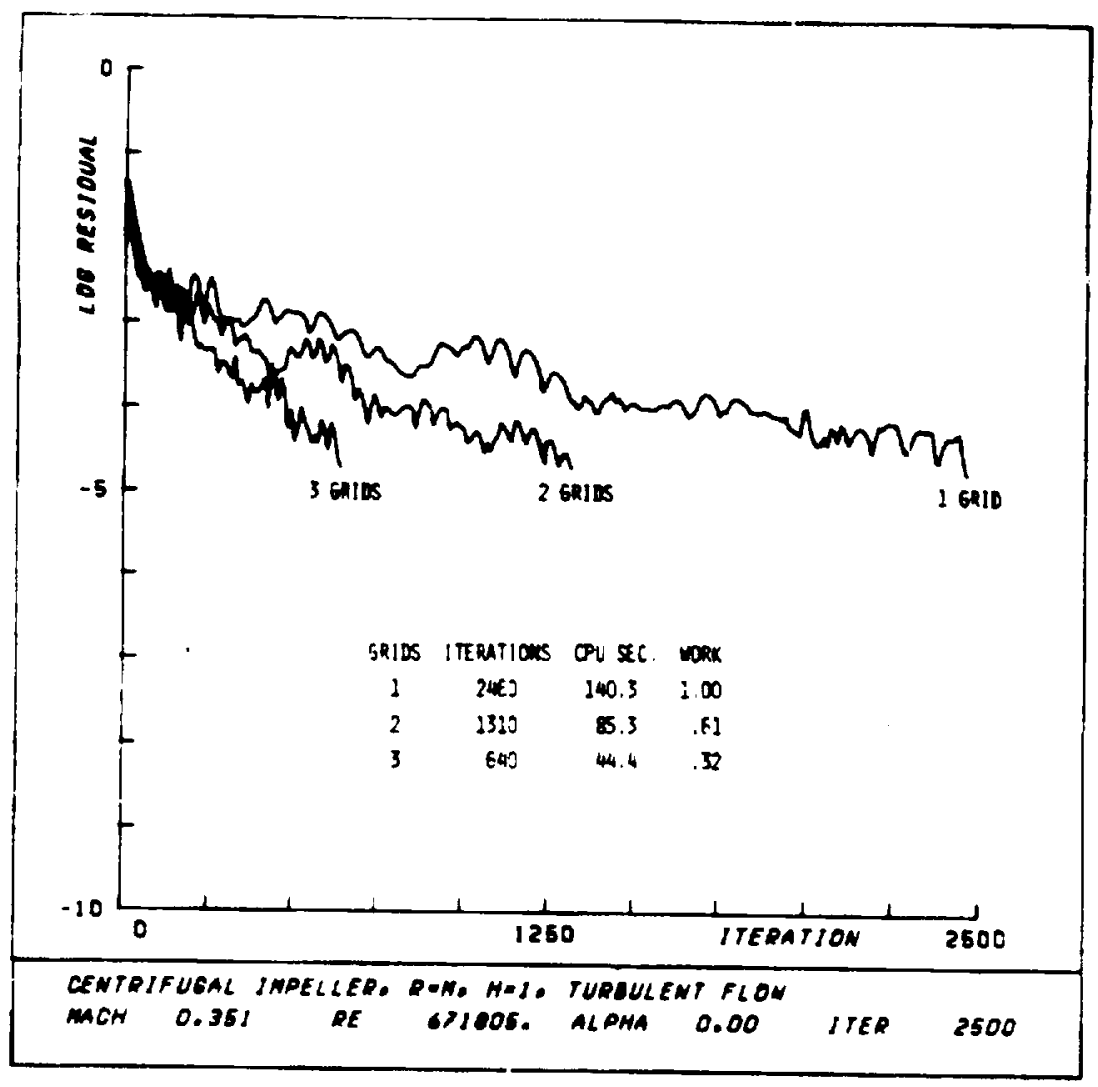

Figure 7. - Multigrid convergence histories for turbulent centrifugal impeller model.

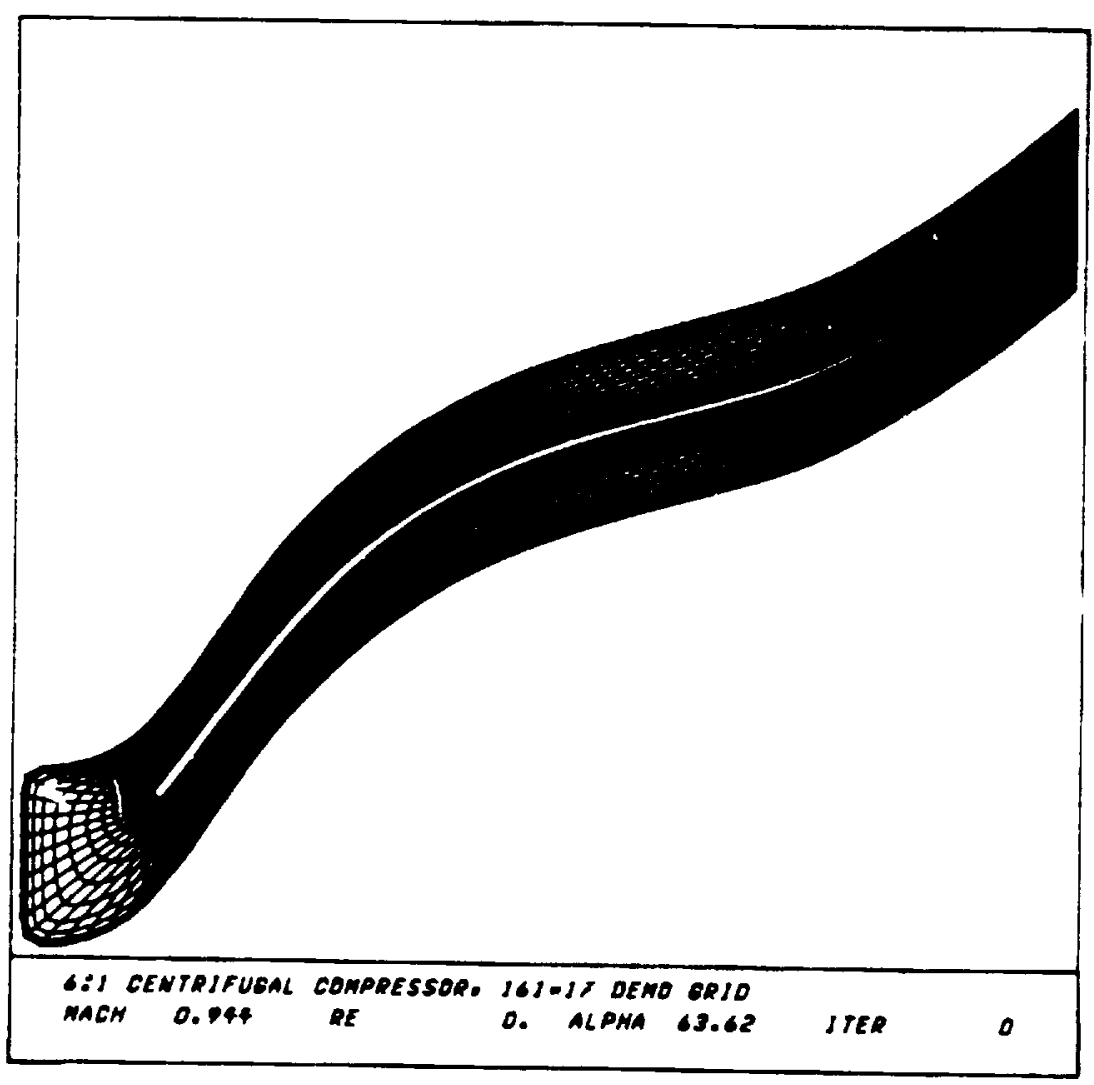

Figure 8. - Computational grid for 6:1 pressure ratio centrifugal impeller.

it 


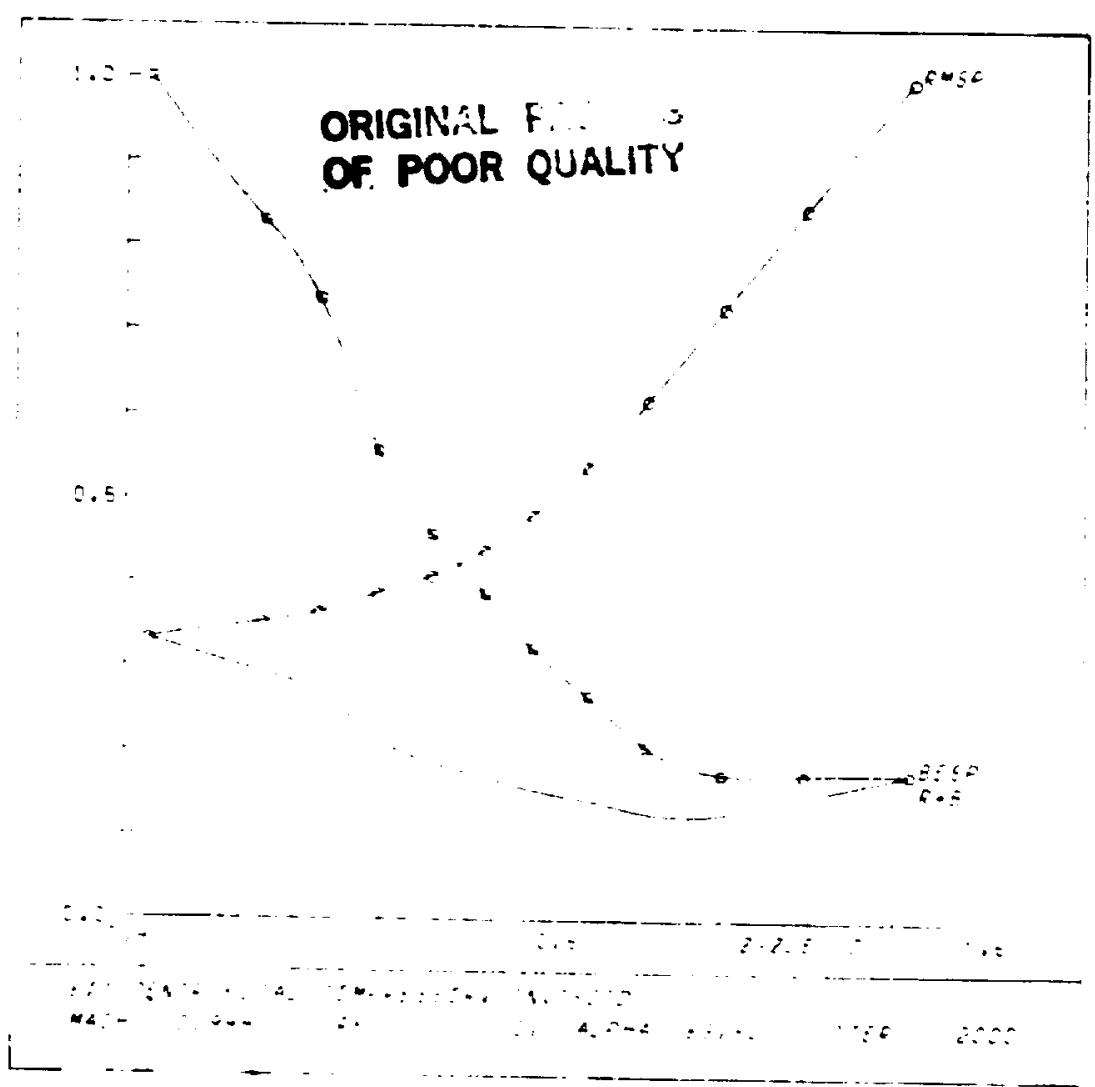

Figure 9. - Radius (RMSP) and stream surface thickness (BESP) for 6:1 centrifugal impeller.

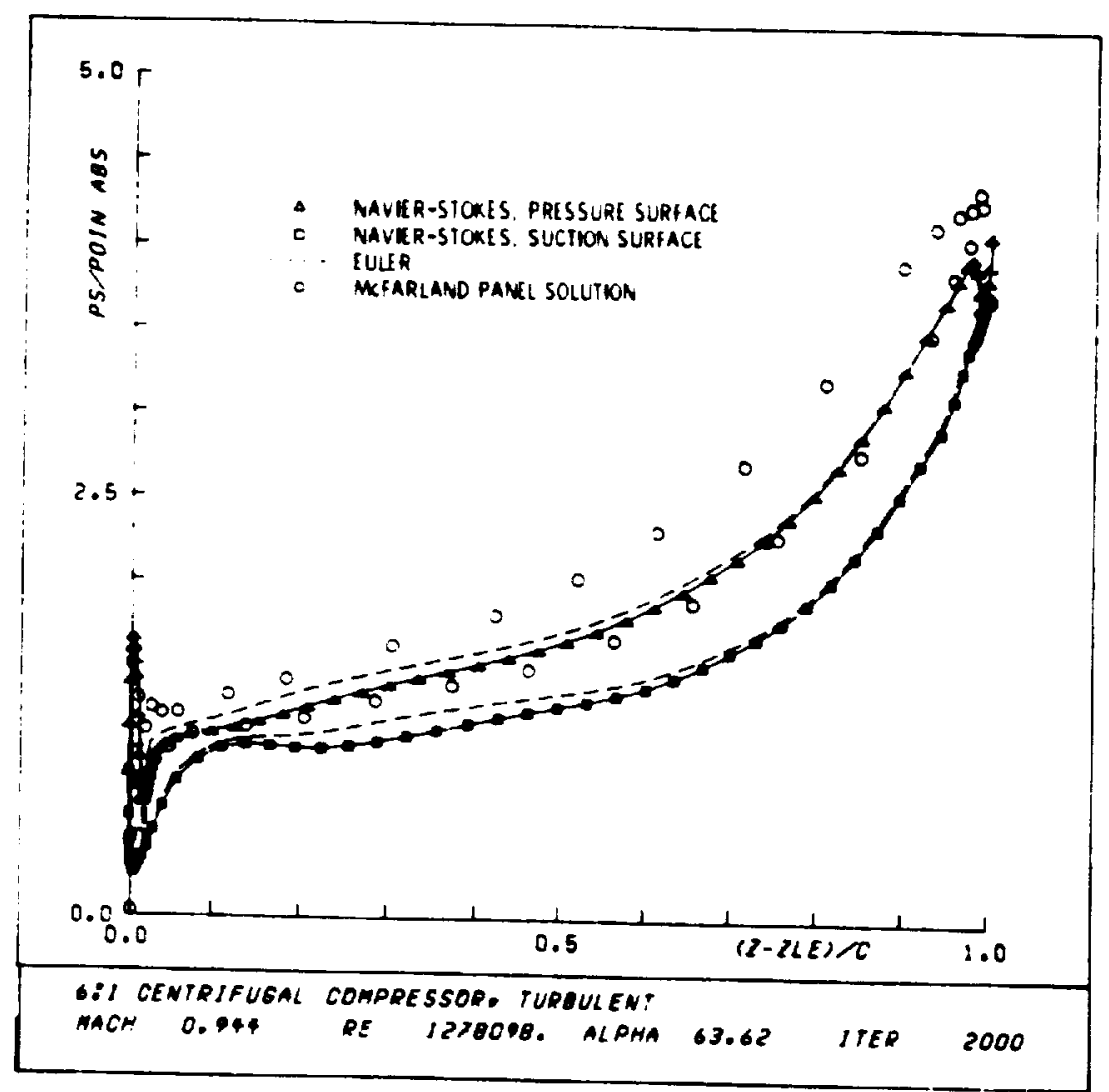

Figure 10. - Static pressure distribution for 6:1 centrifugal impelitr. . 


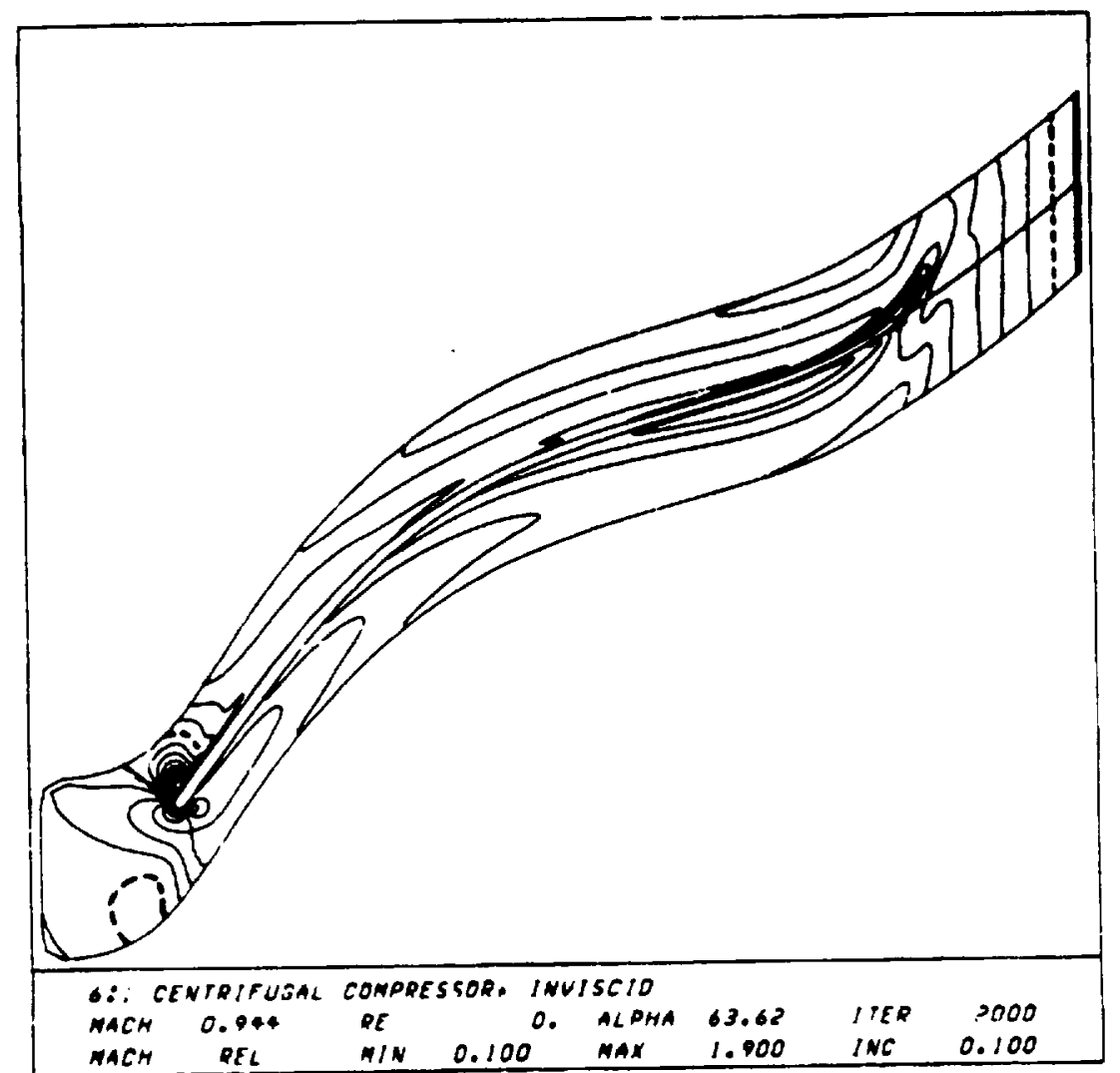

Figure 11. - Mach number contours for inviscid flow in 6:1 centrifugal impeller.

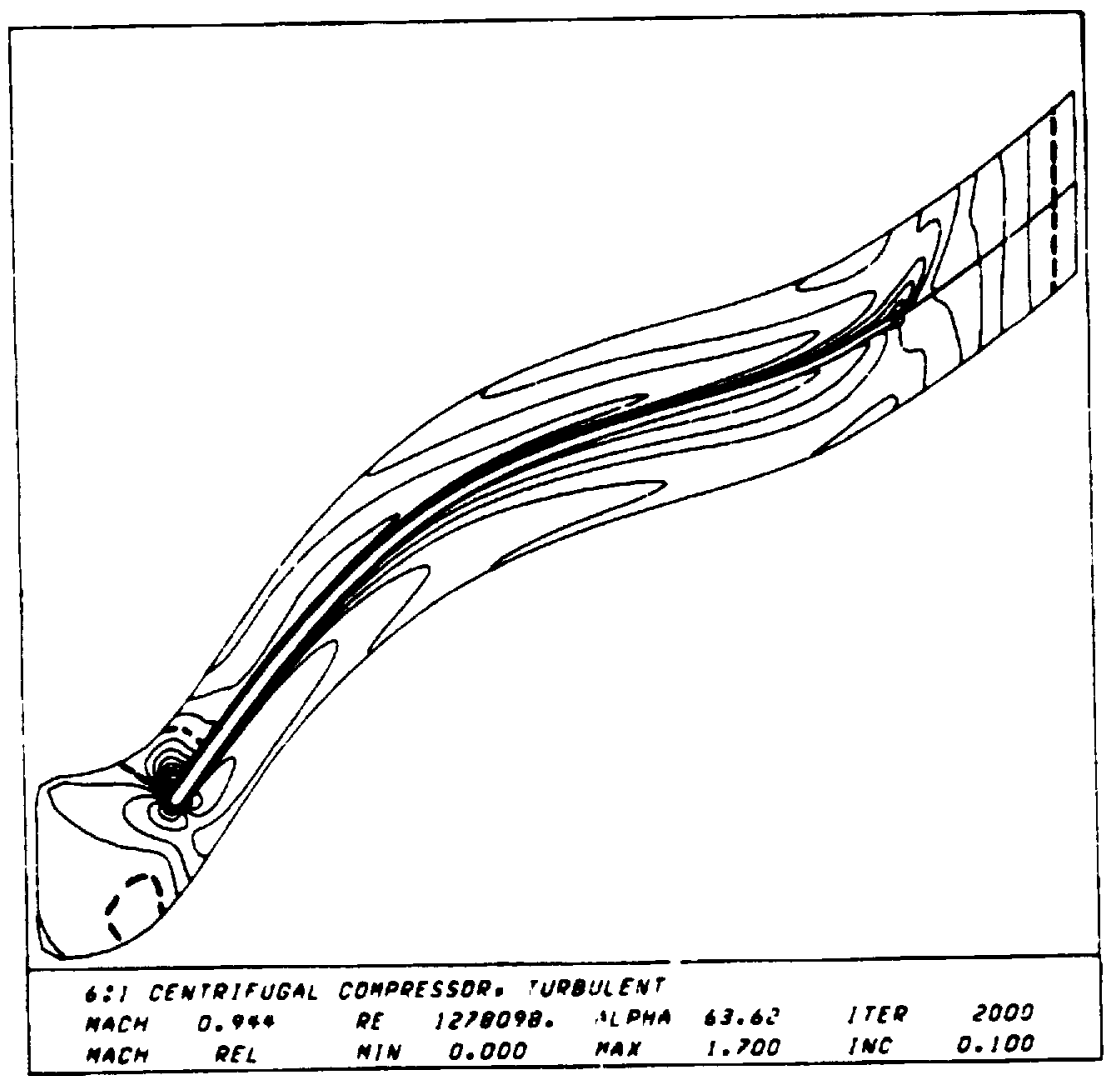

Figure 12 - Mach number contours for turbulent flow in 6:1 centrifugal impeller. 


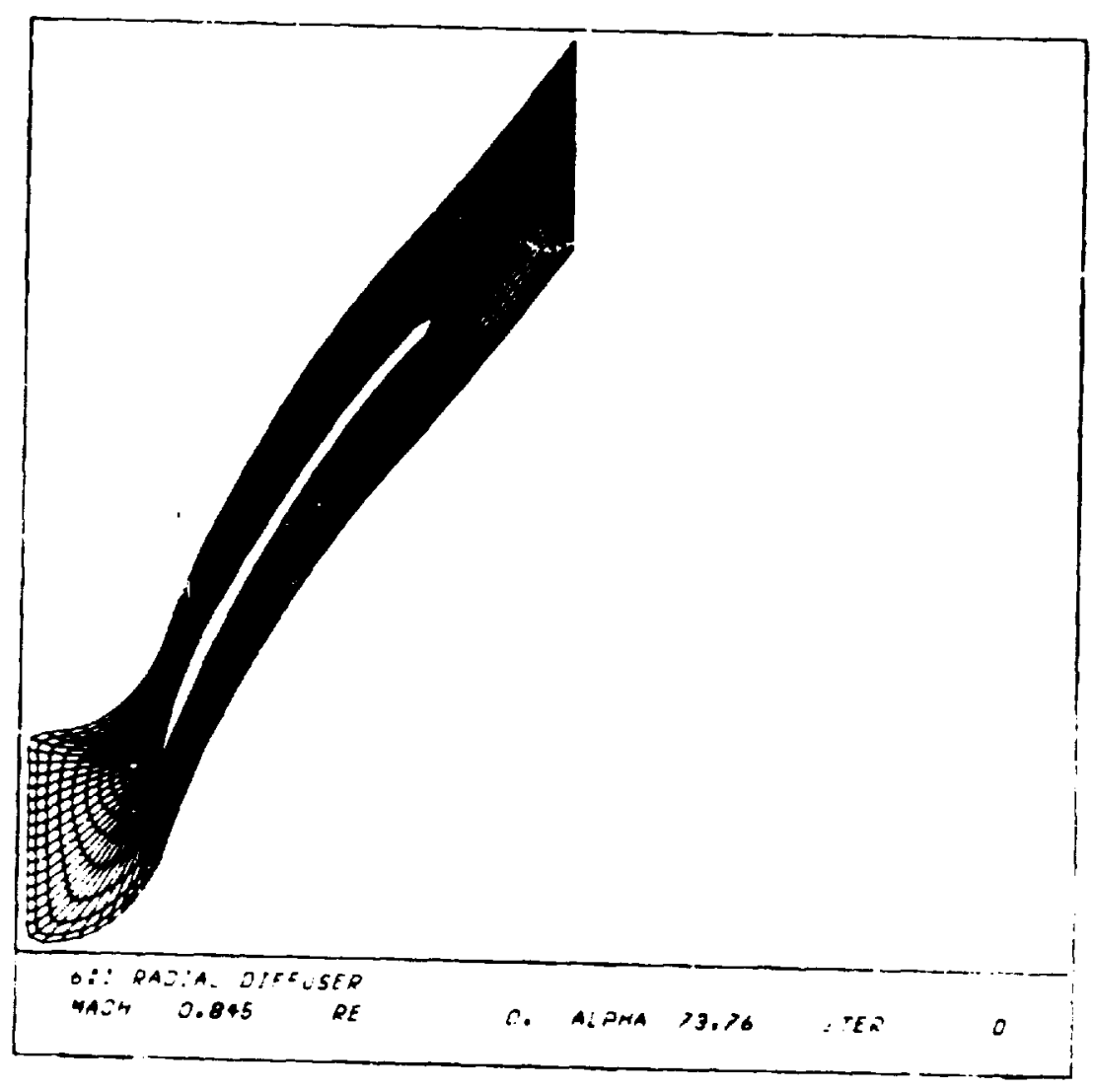

Figure 13. - Computational grid for 6:1 pressure ratio radial diffuser.

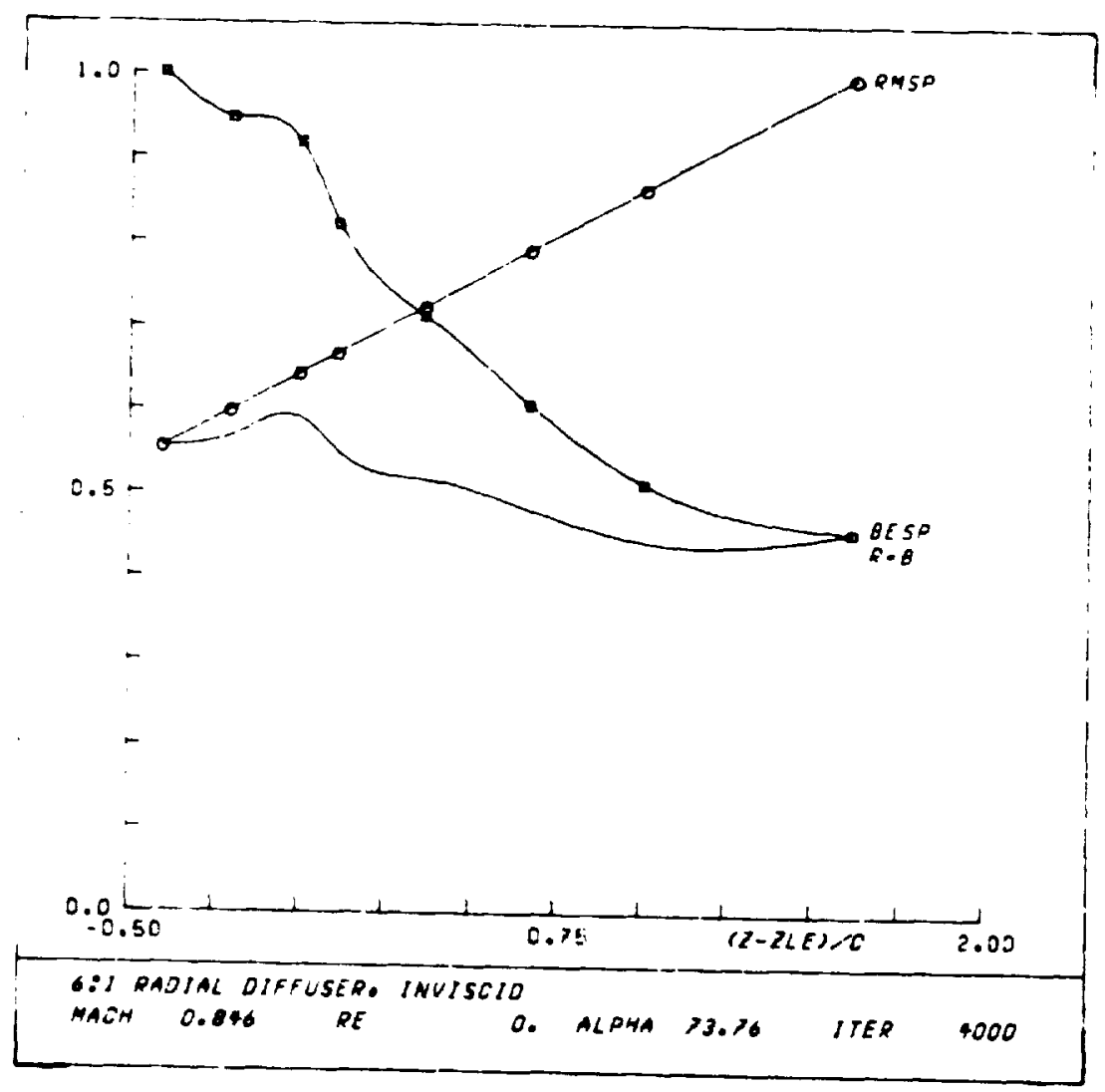

Figure 14. - Radius (RMSP) and stream surface thickness (BESP) for 6:1 radial diffuser.

1 I 


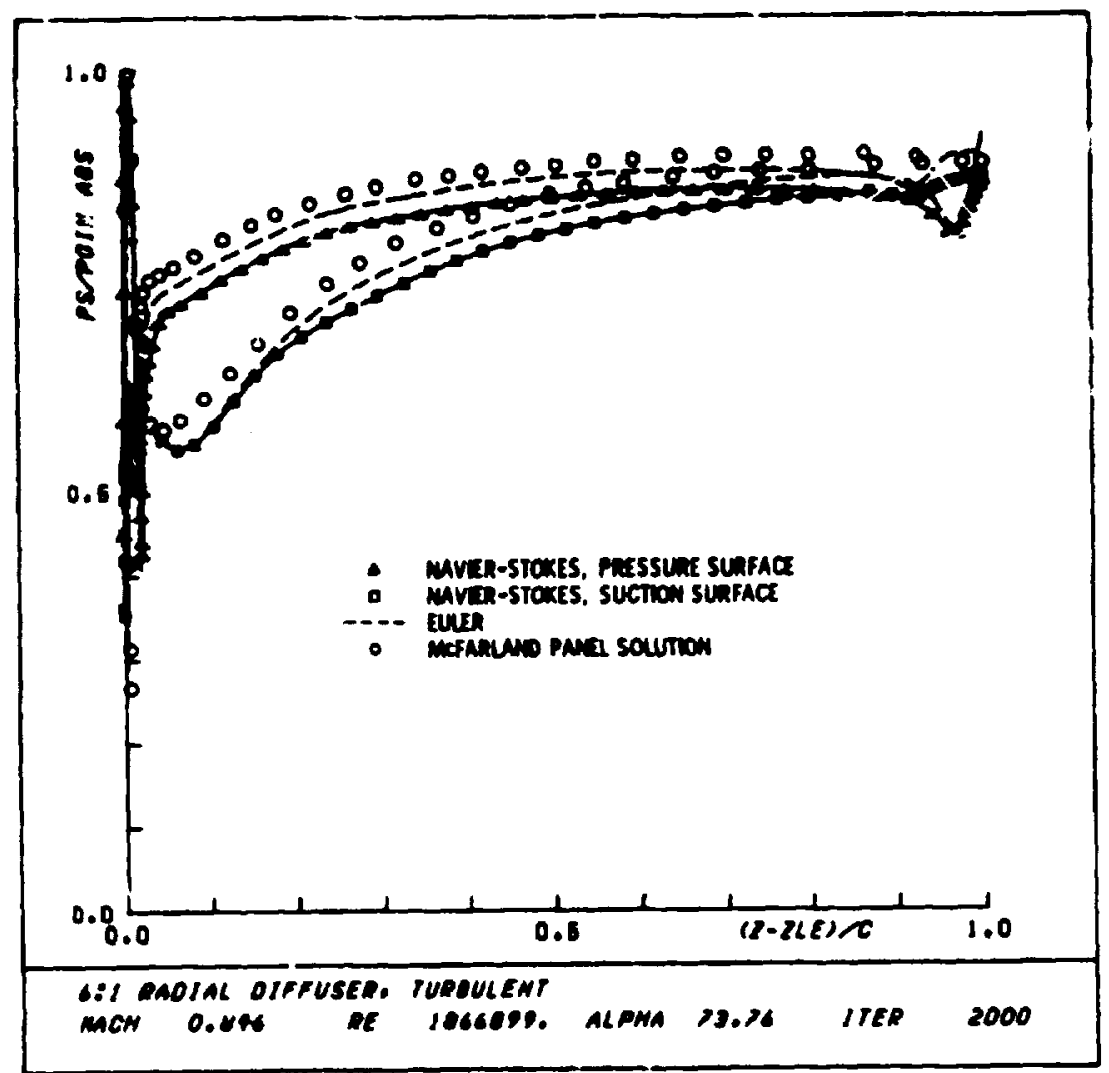

Figure 15. - Static pressure distribution for 6:1 radial dilfuser.

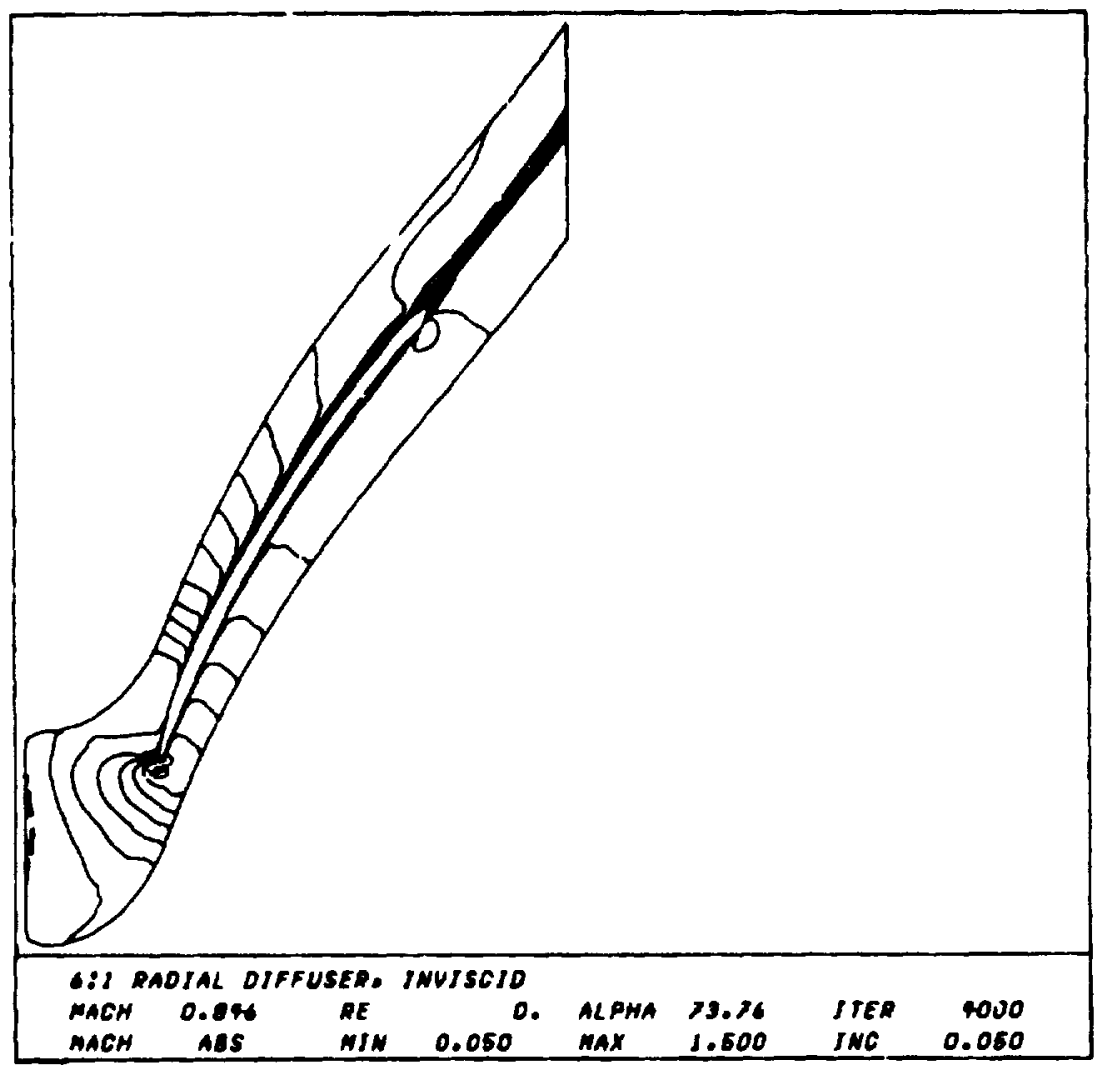

Flgure 16. - Mach number contburs tor Inviscid flow in 6:1 redial diffuser. 


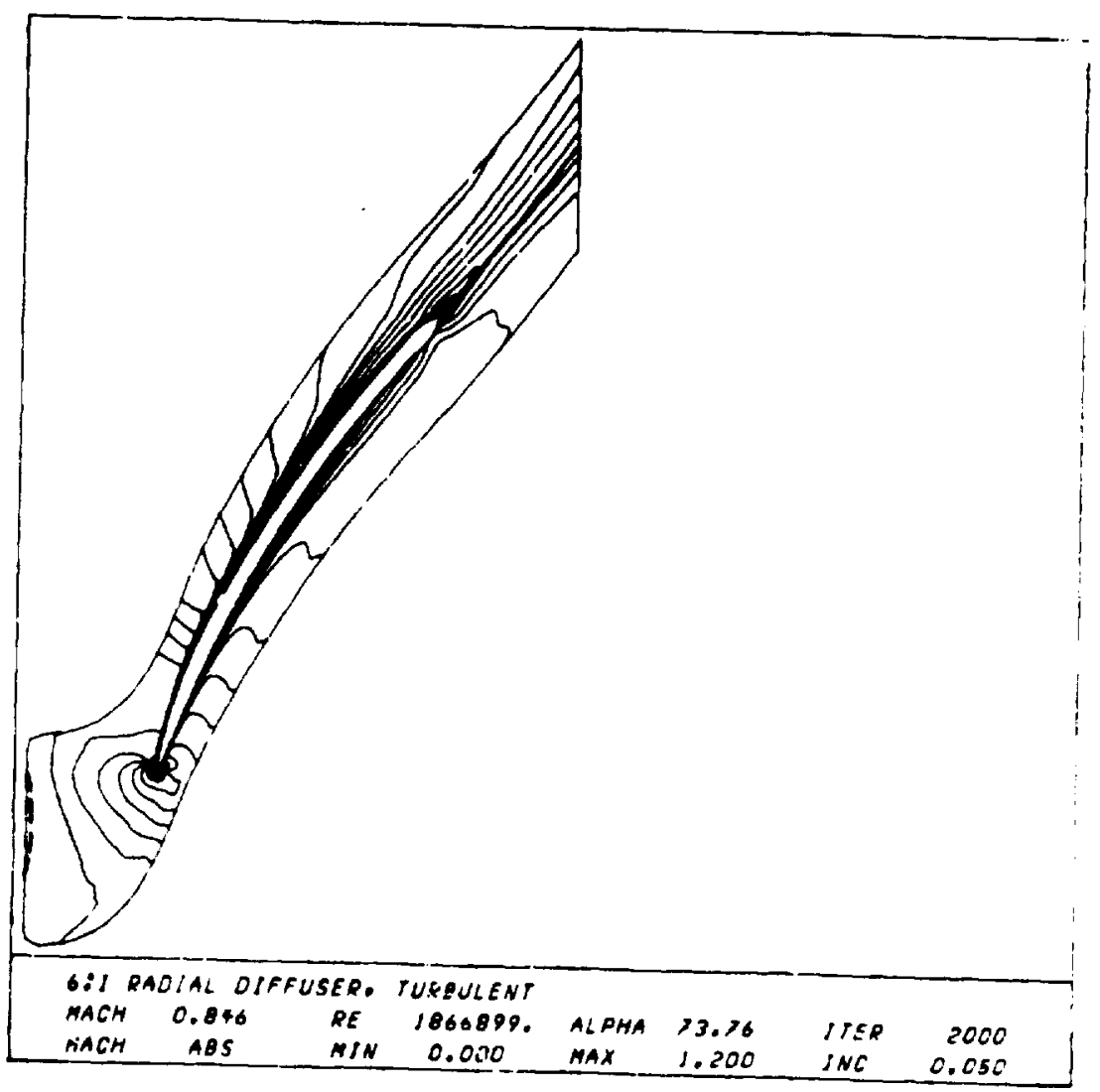

Figure 17. - Mach number cuntours for turbulent flow in 6:1 radial dliiuser. 


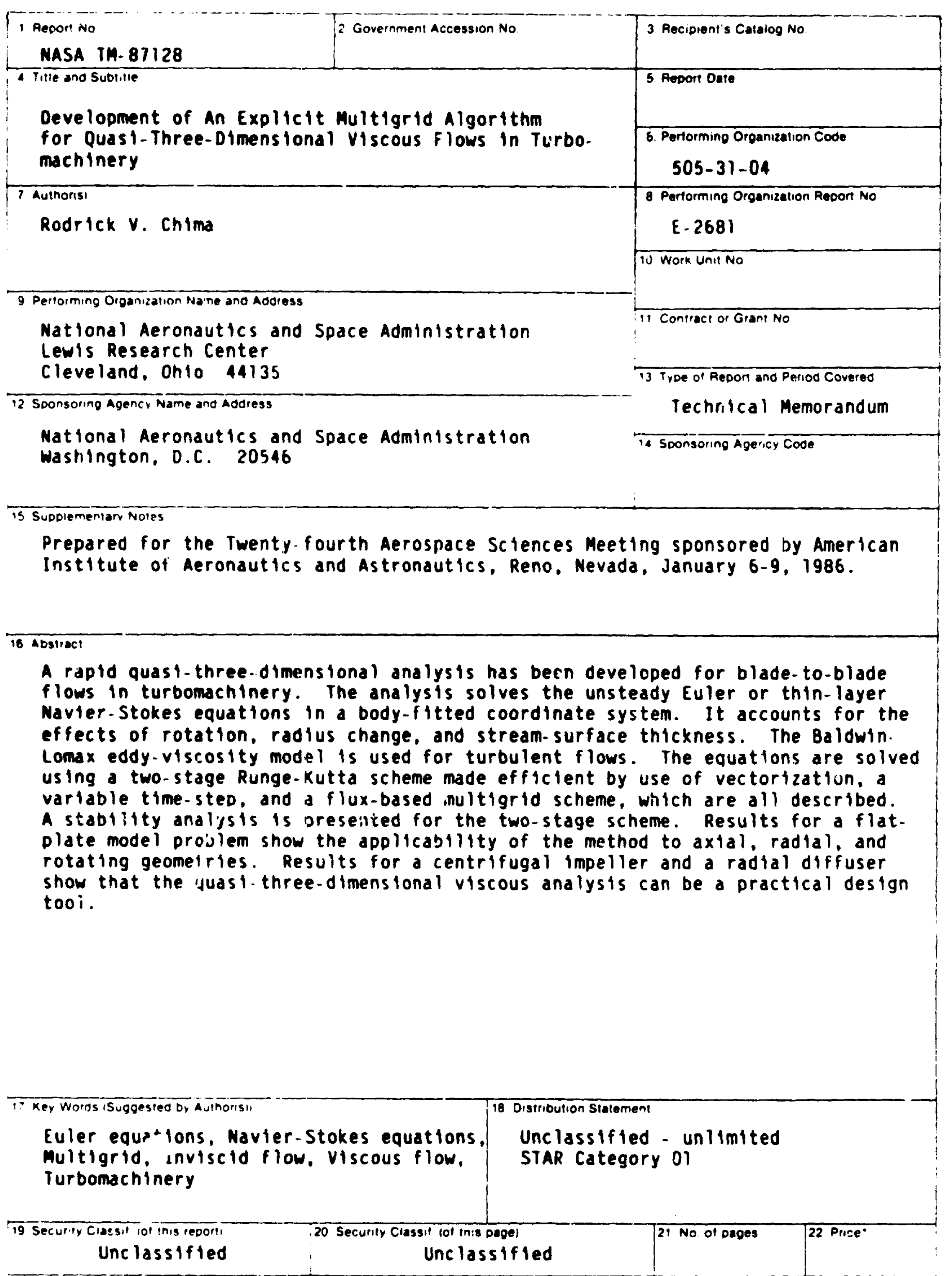


